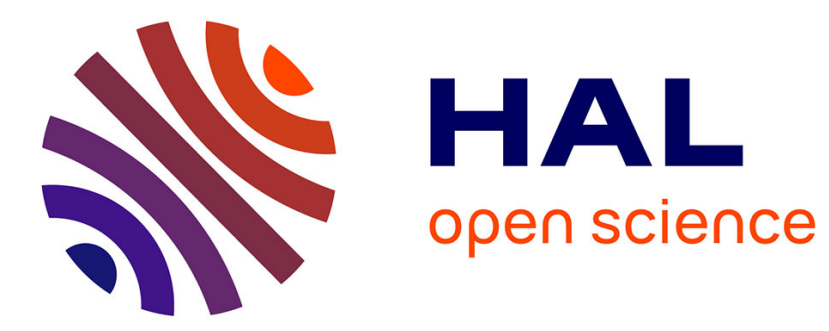

\title{
Techniques de décor de la céramique amathousienne archaïque
}

Floréal Daniel, Sabine Fourrier, Aude Plantey

\section{To cite this version:}

Floréal Daniel, Sabine Fourrier, Aude Plantey. Techniques de décor de la céramique amathousienne archaïque. Bulletin de Correspondance Hellenique, 2007, 131-1, pp.37-65. 10.3406/bch.2007.7455 . hal-01451530

\author{
HAL Id: hal-01451530 \\ https://hal.science/hal-01451530
}

Submitted on 1 Feb 2017

HAL is a multi-disciplinary open access archive for the deposit and dissemination of scientific research documents, whether they are published or not. The documents may come from teaching and research institutions in France or abroad, or from public or private research centers.
L'archive ouverte pluridisciplinaire HAL, est destinée au dépôt et à la diffusion de documents scientifiques de niveau recherche, publiés ou non, émanant des établissements d'enseignement et de recherche français ou étrangers, des laboratoires publics ou privés. 



$$
\begin{gathered}
\text { ÉCOLE FRANÇAISE D'A THÈ NES } \\
\text { B ULLETIN } \\
\text { DE CORRESONDANCE- } \\
\text { HELLÉNIQUE }
\end{gathered}
$$

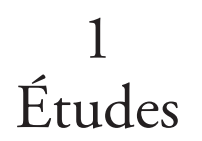

$\mathrm{BCH}$

$\frac{131}{2007}$


É C O L E FR A N ÇA I S E D'A T H È N E S

\author{
B U L L E T I N
}

DE CORRESPONDANCE-
HELLÉNIQUE

\title{
131.12007
}

Comité de rédaction : Dominique MULLIEZ, directeur

Catherine AUBERT, adjointe aux publications

Comité de lecture du BCH 131.1 (2007) :

Roland ETIEnNe

Bernard HoltzManN

Claude Rolley

Eva SimANTONI-BOURNIA

\section{Révision}

et mise au point des textes : Béatrice Detournay

Conception et réalisation : Velissarios Anagnostopoulos, Break In

Coordination de la fabrication: Velissarios Anagnostopoulos

Photogravure

Impression Reliure : $\quad$ Break In s.a.

(C) École française d'Athènes 2009 6, rue Didotou GR - 10680 Athènes www.efa.gr

Dépositaire : De Boccard Édition-Diffusion 11, rue de Médicis F-75006 Paris www.deboccard.com

ISBN 978-2-86958-208-8

Reproduction et traduction, même partielles, interdites sans l'autorisation de l'éditeur pour tous pays, y compris les États-Unis. 


\section{SOMMAIRE DE LA LIVRAISON}

\section{I. Études}

Laurence AlPE, Thierry PetrT et Gilles VelHo

Sondage stratigraphique au palais d'Amathonte en 1997.

Nature et chronologie du premier état

Floréal Daniel, Sabine Fourrier, Aude Plantey et Agnès Rôhfritsch

Techniques de décor de la céramique amathousienne archaïque

Sabine FoURRIER

Le dépôt archaïque du rempart Nord d'Amathonte, V.

Céramiques culinaires

Thomas BRISART

L'atelier de pithoi à reliefs d'Aphrati. Les fragments du musée Bénaki

Heide Frielinghaus

Die Helme von Delphi

Myriam FinCKer et Jean-Charles MORETTI

Le barrage du réservoir de l'Inopos à Délos

Hélène SIARD

Dédicace d'un mégaron dans le Sarapieion C de Délos.

Cédric Brélaz, Angheliki K. Andreiomenou et Pierre Ducrey

Les premiers comptes du sanctuaire d'Apollon à Délion

et le concours pan-béotien des Delia

Julien Fournier et Patrice HAMON

Les orphelins de guerre de Thasos:

un nouveau fragment de la stèle des Braves (ca 360-350 av. J.-C.)

Alexandre Avram, Costel Chiriac et Ionel Matei

Defixiones d'Istros

Athanasios Tziafalias et Bruno Helly

Décrets inédits de Larissa (3) $421-474$ 


\section{Yannis KALLIONTZIS}

Décrets de proxénie et catalogues militaires de Chéronée trouvés lors des fouilles de la basilique paléochrétienne d'Haghia Paraskévi

Sandrine ELAIGNE

La circulation des céramiques fines hellénistiques dans la région égéenne :

un aperçu à partir du mobilier de Délos et de Thasos

\section{Christophe FlaMENT}

Die et engraver-sharing dans le Péloponnèse

entre le règne d'Hadrien et celui de Septime

Jean-Michel SAULNIER

Le trésor de monnaies médiévales de Potamia (Chypre)

$615-719$

Jean-Pierre De RYCKE

Arnould de Vuez, "Peintre flamand " du marquis de Nointel et les premiers dessins du Parthénon en 1674 
ÉTUDES 



\title{
Techniques de décor de la céramique amathousienne archaïque*
}

\author{
Floréal DANIEL, Sabine Fourrier, Aude Plantey et Agnès RÖHFritsCH
}

RÉSUMÉ Fruit d'une collaboration entre l'EFA et l'UMR 5060 du CNRS, le programme d'étude de la céramique amathousienne archaïque porte sur différents aspects de l'artisanat, depuis l'exploitation des matières premières jusqu'aux techniques de fabrication des vases.

Cet article traite des modes de décoration, concernant trois procédés typiquement amathousiens : les fabriques "Blackened ", le "Bichrome amathousien " et l'imitation de la technique de la figure noire. Les analyses scientifiques mettent en relief le savoir-faire des potiers et des peintres amathousiens qui, en jouant sur quelques techniques simples, et notamment les modes de cuisson, ont su renouveler le répertoire chypriote sans jamais s'affranchir des procédés de fabrication traditionnels.

ПЕРІ

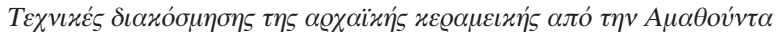

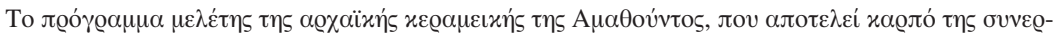

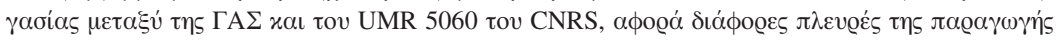

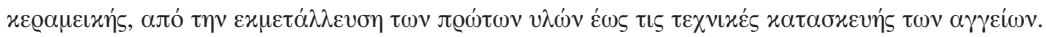

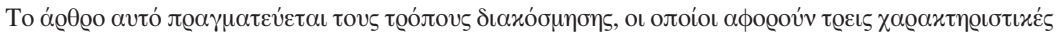

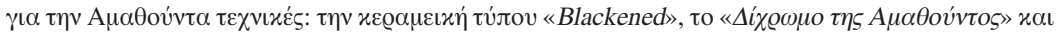

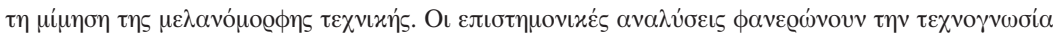

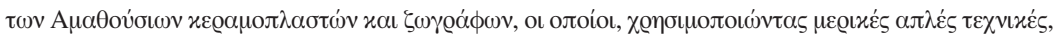

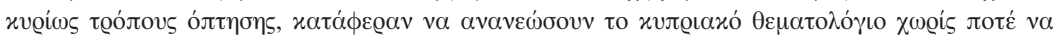

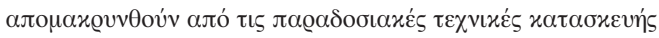

\section{Decorative Techniques in the Archaic Pottery of Amathus}

Fruit of collaboration between EFA and UMR 5060 of CNRS, the programme of study of the ancient Amathusian pottery concerned various aspects of the craft, from the use of raw materials to the techniques for making vases.

This article deals with methods of decoration, specifically three typically Amathusian styles: 'Blackened', 'Bichrome Amathusian' and an imitation of the black-figure technique. The scientific analyses throw into relief the expertise of Amathusian potters and painters who, by playing with a number of simple techniques, notably firing methods, successfully renewed the Cypriot repertoire without breaking free of the traditional manufacturing methods. 
La classification de la céramique chypriote de l'Âge du Fer, publiée par E. Gjerstad en 1948 , repose, pour l'essentiel, sur les modes de décoration ${ }^{1}$. Trois grandes catégories sont définies selon que le décor est appliqué sur une surface claire, engobée ou non, sur une surface portant une couverte rouge ou sur une surface portant une couverte noire. Chacune de ces catégories est subdivisée en Wares (que nous traduisons par «fabriques »), toujours en fonction du décor : par exemple, la première catégorie comprend les fabriques Plain White (vases à surface claire sans décor peint), White Painted (vases à surface claire portant un décor peint monochrome, généralement noir) et Bichrome (vases à surface claire portant un décor peint en deux couleurs différentes, généralement noir et rouge). L'évolution des formes et du répertoire décoratif permet d'ordonner dans le temps chacune des fabriques, selon un découpage en sept classes successives, de la période géométrique à la fin de l'époque classique : par exemple, le White Painted I apparaît dès le Chypro-Géométrique I et la fabrique est attestée tout au long de l'Âge du Fer ; le Blackon-Red I(III), en revanche, n’apparaît qu'au Chypro-Géométrique III, les céramiques à engobe rouge n'étant produites dans l'île qu'à partir de cette date. Le système est simple et

* Cet article présente un premier état des résultats du programme de recherche sur la céramique amathousienne archaïque, obtenus dans le cadre d'une collaboration entre l'École française d'Athènes et le Centre de Recherche en Physique Appliquée à l'Archéologie (UMR 5060 du CNRS). Cette étude fait partie des programmes qui bénéficient d'un co-financement du CNRS dans le cadre de la convention signée avec l'École française d'Athènes. Ont également participé à ces travaux S. Nougaret, N. Cadeillan et M. Videau. Nous remercions vivement Annie Caubet de l'autorisation de reproduire des vases conservés au musée du Louvre, et Catherine Giraudon, qui nous a procuré les tirages.

Tous nos remerciements vont également au Département des Antiquités de Chypre et à son directeur, Pavlos Flourentzos, qui nous a généreusement autorisés à exporter les échantillons.

Les abréviations utilisées sont les suivantes :

CVA = Corpus Vasorum Antiquorum.

Hermary 1986 = A. Hermary, "Divinités chypriotes II ", RDAC, p. 164-172, pl. XXXIV-XXXV.

KARAgEORGHIS 1982 = V. KarageOrGHIS, "Black Slip Grooved Ware from Cyprus », RDAC, p. 119-122, pl. XXI-XXII.

KARAgEOrghis 1989 = V. KARAgEORghis, "Some Remarks on the "Amathus Style" in Cypriote VasePainting ", in H.-U. Cain, H. Gabelmann, D. Salzmann (éds), Festschrift für Nikolaus Himmelmann, p. 83-86.

KARAgEORGHIS, des GaGNIERS 1974 = V. KARAGEORGHIS, J. des GAGNIERS, La céramique chypriote de style figuré.

Karageorghis, Laffineur, Vandenabeele 1997 = V. Karageorghis, R. Laffineur, Fr. Vandenabeele (éds), Four Thousand Years of Images on Cypriote Pottery. Proceedings of the Third International Conference of Cypriote Studies, Nicosie, 3-4 May 1996.

SCE IV/2 = E. GJERSTAD, The Swedish Cyprus Expedition. Finds and Results of the Excavations in Cyprus 19271931, IV. 2. The Cypro-Geometric, Cypro-Archaic and Cypro-Classical Periods (1948).

1. SCE IV/2, p. 48-91. 
efficace : valable pour l'ensemble de l'Âge du Fer, il l'est aussi pour l'ensemble des régions de l'île².

Cette classification générale ne rend toutefois pas compte des spécificités régionales. Or, après une période géométrique caractérisée par une profonde uniformité des productions, des originalités, des tendances, morphologiques et décoratives, naissent dans les ateliers des différents royaumes chypriotes. Le phénomène est très sensible à Amathonte où, dès le début de la période archaïque ${ }^{3}$, les artisans définissent, en adoptant de nouvelles formes et de nouveaux motifs décoratifs, un style amathousien particulièrement inventif. Cette créativité, qui va en s'accentuant au cours de la période, touche les formes : cruches à embouchure trilobée et panse globulaire, très " écrasée " (fig. 1), quand les ateliers de Salamine, par exemple, privilégient des formes plus élancées ${ }^{4}$; amphores de grande taille à col court et anses épaisses du col à l'épaule (fig. 2$3)^{5}$. Elle est également perceptible dans les choix et la réalisation des motifs décoratifs. Le répertoire amathousien échappe à la stricte division de la céramique chypriote archaïque en deux grandes " provinces stylistiques ", l'une de tradition géométrique, l'autre figurée, esquissée par E. Gjerstad en $1960^{6}$ : comme les ateliers de l'Est de l'île, ceux d'Amathonte sont friands de motifs figurés, notamment d'oiseaux qui déploient librement leurs ailes sur la panse des cruches, mais ils les rendent de manière géométrique, efficace, mais sèche (fig. 1) ; s'ils savent, à l'occasion, composer des scènes plus ambitieuses, les peintres amathousiens les coincent sur l'épaule du vase, tandis que les frises de cercles concentriques, chères aux ateliers occidentaux, occupent l'essentiel de la panse (fig. 2-3). Cette originalité se manifeste enfin dans les techniques de décor qui, sans jamais s'affranchir du carcan des fabriques traditionnelles, déclinent des variations sur des schémas connus. Certaines de ces particularités techniques ont déjà été soulignées : délaissé par les autres ateliers de l'île, le Black Slip d'époque géométrique est repris à

2. Même si certaines fabriques sont plus en vogue dans certains ateliers que dans d'autres : le Black-on-Red est ainsi beaucoup plus commun dans la région de Paphos que dans celle de Salamine, par exemple. Cf. N. SCHreiber, The Cypro-Phoenician Pottery of the Iron Age, Culture and History of the Ancient Near East 13 (2003).

3. On peut en repérer les racines dès la fin de la période géométrique : Fr. VANDENABEELE, "Quelques particularités de la civilisation d'Amathonte à l'époque chypro-géométrique ", $B C H 92$ (1968), p. 103-114, pl. XIV-XVI.

4. Comparer avec SCE IV/2, fig. XXXIV, 1-4 (Bichrome IV, type 14).

5. A. Hermary, O. Masson, "Deux vases inscrits du sanctuaire d'Aphrodite à Amathonte (1865-1987)", $B C H 114$ (1990), p. 187-214. L'amphore publiée, exceptionnelle en raison du décor figuré et de l'inscription qu'elle porte, entre dans une série de production, documentée par d'autres exemplaires, provenant du même contexte (grotte votive du sanctuaire d'Aphrodite) et d'un dépôt palatial, fouillé plus récemment contre le rempart Nord de la ville basse.

6. E. GJeRSTAD, « Pottery Types, Cypro-Geometric to Cypro-Classical », OAth 3 (1960), p. 105-106. 
Amathonte, sous impulsion levantine, dans un genre nouveau ${ }^{7}$; combinant techniques de décor sur surface claire et sur surface engobée, les artisans amathousiens rompent la stricte séparation des fabriques et créent un style hybride (fig. 4) ${ }^{8}$. Mais il manquait une étude d'ensemble qui envisageât toute la céramique amathousienne archaïque, et non pas seulement les séries originales, dans son cadre de production.

Des observations au microscope et des analyses physico-chimiques, en mettant en évidence les ressorts techniques du style amathousien, démontrent à la fois l'inventivité et la cohérence du savoir-faire des artisans. Ces derniers ont su renouveler le répertoire de tradition chypriote, en conciliant innovations et traditions et en jouant sur les mêmes procédés techniques, qu'il s'agisse des fabriques "Blackened ", du "Bichrome amathousien » ou de l'imitation de la technique de la figure noire d'origine grecque.

\section{LES FABRIQUES «BLACKENED ${ }^{9} »$}

La technique de décoration avec couverte noire, dite "Black Slip» dans la terminologie suédoise, est attestée dans l'île dès le début de l'Âge du Fer. Les vases, de forme fermée ou ouverte, sont recouverts d'un engobe noir, appliqué sur une surface à godrons verticaux ${ }^{10}$. Cette surface engobée ne porte jamais de décor peint. À cet égard, les dénominations des variantes de la fabrique, forgées par E. Gjerstad, "Black Slip Painted " et "Black Slip Bichrome ${ }^{11}$ ", sont trompeuses. De fait, il s'agit, à proprement parler, de fabriques hybrides, la surface d'un même vase étant en partie engobée (Black Slip) et en partie réservée, avec un décor géométrique peint en noir (White Painted) ou en noir et rouge (Bichrome). Dès la fin de la période géométrique, la production des variantes hybrides est abandonnée, les godrons tendent à disparaître et le Black Slip n'est plus utilisé que pour des formes très simples, le plus souvent des cruches. La fabrique, dont l’apogée est à placer au Chypro-Géométrique I (ca 1050-950 av. J.-C.), est définitivement supplantée à partir du Chypro-Géométrique III (ca 900-750) par les fabriques à couverte rouge (Red Slip et variantes à décor peint, Black-on-Red et Bichrome Red). Elle est très peu utilisée au cours de l'époque archaïque, toujours pour des vases de morphologie simple et de réalisation

7. La fabrique a été identifiée par V. Karageorghis, qui l’a dénommée "Black Slip Grooved Ware " (KARAGEORGHIS 1982).

8. L. Alpe, S. Fourrier, "Une production originale d'Amathonte : les amphores à anses horizontales et les dinoi de style hybride », CCEC 33 (2003), p. 149-167.

9. Nous remercions K. Nys, qui nous a suggéré d'employer ce terme, davantage conforme à la réalité de la technique utilisée que les appellations en "Black Slip».

10. SCE IV/2, fig. IX-X, XVII, XXVI.

11. Ibid., fig. IX et XVI. 
peu soignée (fig. 5). Elle ne connaîtra un regain tardif qu'au cours de l'époque classique, sous l'influence du vernis noir attique, influence que les formes (vases à boire et plats) trahissent ${ }^{12}$.

Il est donc étonnant de remarquer, dans la production amathousienne archaïque, dès le Chypro-Archaïque I ( $c$ 750-600) et plus encore à partir du Chypro-Archaïque II (ca 600-475), un essor des fabriques à couverte noire. Ces dernières sont à distinguer du "Black Slip Grooved Ware » qui, comme son nom l'indique, est caractérisé par l'application d'un engobe noir sur une surface à godrons horizontaux et qui n'occupe, d'ailleurs, qu'une place anecdotique dans la production amathousienne ${ }^{13}$. À la différence du Black Slip géométrique, les vases archaïques portent un décor peint (en noir ou en noir et blanc) directement appliqué sur la couverte noire et non sur une zone réservée, à côté de cette dernière. Enfin, d'un point de vue typologique et stylistique, ces vases présentent un équivalent exact, en version " noircie » ou, pour garder l'anglais cher à la terminologie suédoise, "Blackened", des productions à surface rouge (Black-on-Red et Bichrome Red), qu'elles tendent même à remplacer vers la fin de la période (fig. 6-7) ${ }^{14}$. La liaison entre les deux séries de production est d'ailleurs soulignée par l'existence de bols hybrides, portant un décor sur couverte rouge à l'intérieur et noire à l'extérieur, ou vice-versa.

L'identification de ces fabriques comme Black Slip est assurément à exclure. Le terme même de Black Slip est à éviter dans leur dénomination, tant ces productions ne doivent rien à la tradition géométrique ${ }^{15}$. La quantité et la qualité des vases invitent, par ailleurs, à ne pas les interpréter comme des accidents de cuisson. Toutes ces hypothèses ont été confirmées par les observations au microscope et les analyses physico-chimiques menées sur neuf échantillons représentatifs de cette production, provenant du dépôt du rempart

12. Ibid., fig. LXI (Black Slip VI).

13. Aux sept exemples recensés par KARAGEORGHIS 1982, on ajoutera quatre vases : le premier provient d'une tombe de Limassol-Agios Athanasios (P. Flourentzos, "The Tombs 214-215 from Ayios Athanasios, Limassol ", RDAC 1993, pl. XXIX, 81) ; deux autres, de provenance inconnue, sont conservés au Royal Museum d'Ontario (V. Karageorghis, Ancient Cypriote Antiquities in the Royal Ontario Museum [2003], $\mathrm{n}^{\circ} 74$ ) et au musée national d'Athènes (id., Ancient Cypriote Art in the National Archaeological Museum of Athens [2003], no 142) ; le dernier, qui aurait été trouvé à "Andimou " (= Avdimou, village à l'Ouest de Kourion ?), est aujourd'hui au musée du Louvre (CVA France 5, Louvre 5, pl. 27, 1 et 3). Parmi les énormes dépôts de céramique archaïque fouillés par la mission française à Amathonte, seuls quelques tessons de panse, recueillis dans la grotte votive du sanctuaire d'Aphrodite, appartiennent à une cruche, réalisée dans cette fabrique.

14. Le procédé a été bien observé par E. Gjerstad, qui ne le note toutefois que pour la fabrique Bichrome Red II(V), sans en souligner la fréquence (SCE IV/2, p. 74).

15. Elles avaient été dénommées "Black Slip-Black-on-Red» et "Black Slip à peinture blanche " par Fr. VANDENABEELE, "Un dépôt de céramique archaïque chypriote dans un silo à Amathonte ", BCH 109 (1985), p. 631. 
Nord (fig. 8). Les tessons, recueillis dans un contexte du Chypro-Archaïque II, proviennent d'amphorisques et de bols Bichrome Red «Blackened ». Parmi ces derniers, certains présentent une face externe rouge ou noire différente de la face interne (noire ou rouge).

Les observations des coupes épaisses polies en lumière naturelle, cathodoluminescence $^{16}$ et par imagerie au microscope électronique à balayage (MEB) ${ }^{17}$ ont permis d'étudier la texture des échantillons (porosité, taux de dégraissant). La composition élémentaire de la matrice de terre cuite et celle des inclusions ont été établies par microanalyse $\mathrm{X}(\mathrm{MEB} / \mathrm{EDS})^{18}$. La nature minérale du dégraissant a été confirmée par microspectrométrie Raman ${ }^{19}$ et par analyse pétrographique sur lames minces.

Le groupe d'échantillons de céramiques "Blackened » est très homogène. La composition élémentaire de la terre cuite est sensiblement identique pour tous les tessons et comparable à celle des autres céramiques archaïques de production amathousienne. Il en est de même pour la nature et la répartition du dégraissant. Tous les échantillons (amphorisques et bols) présentent des inclusions minérales de même nature, caractéristiques de la production locale (chromites et pyroxènes). Seule la proportion de ces inclusions varie d'un objet à l'autre. Les décors noirs sont obtenus grâce à un oxyde de manganèse qui est, lui aussi, un élément caractéristique de la géologie de la région amathousienne.

L'observation de coupes épaisses polies révèle que, sous l'engobe, la terre cuite de ces céramiques a une couleur beige-vert. L'analyse montre que le taux de calcium est important ( 15 à $25 \%$ exprimés en pourcentages $\mathrm{de} \mathrm{CaO}$ ). Or, avec le type d'argile calcaire employée, la décomposition de la calcite et la formation progressive au cours de la cuisson d'alumino-silicates de calcium permet d'évaluer, par diffraction de rayons $\mathrm{X}$, les températures atteintes. En l'occurrence, les diffractogrammes ont révélé des phases cristallines (géhlénite), caractéristiques de températures de cuisson supérieures à $850^{\circ} \mathrm{C}^{20}$.

L'analyse de la composition élémentaire des engobes montre que seul le taux de calcium la différencie de celle de la terre cuite. Les potiers ont donc sans doute employé la même argile. La composition de la terre cuite, plutôt calcaire, et celle des engobes, plutôt

16. La cathodoluminescence (CL) peut être définie comme l'émission d'une lumière visible ou proche du visible provoquée par un bombardement électronique. La couleur obtenue est souvent (mais pas toujours) caractéristique des minéraux et des impuretés que ces derniers contiennent. L'appareillage utilisé comporte une chambre de CL OPEA. L'observation est réalisée à travers un microscope optique Olympus BX51. Une caméra Olympus DP 50 permet la capture des images.

17. Microscope électronique à balayage à pression variable Jeol $6460 \mathrm{LV}$.

18. Microanalyse EDSX Inca 300 de Oxford Instruments.

19. Microspectromètre Raman Renishaw. Laser Helium-Neon à 633 nm.

20. T. Peters, R. Iberg, "Mineralogical Changes During Firing of Calcium-Rich Brick Clays ", American Ceramic Society Bulletin 57/5 (1978), p. 503-509. 
siliceuse, pourraient être dues à l'affinage (lavage et décantation) d'une même argile, afin de ne conserver que la fraction argileuse en diminuant les éléments de dégraissant naturel (calcium, feldspaths, etc.).

En revanche, la divergence des taux de calcium permet d'expliquer la différence de couleur entre terre cuite et engobe. Dans les mêmes conditions de cuisson (atmosphère, durée, température), les réactions chimiques responsables de la formation de composés dits de " haute température " (géhlénite, anorthite, wollastonite) different selon que l'argile est calcaire ou siliceuse.

La terre cuite et l'engobe présentent des concentrations en fer voisines. Cependant, les alumino-silicates de calcium formés au cours de la cuisson de la terre cuite empêchent la formation d'oxydes de fer (hématite) ${ }^{21}$, ce qui explique sa couleur beige. En revanche, la faible teneur en calcite dans les engobes ne permet pas la formation de ces composés qui piègent le fer, et ce dernier peut donner des oxydes comme l'hématite (engobe rouge) ou la magnétite (engobe noir).

Il reste à expliquer comment les potiers ont réussi à obtenir des engobes tantôt rouges, tantôt noirs et, dans certains cas, à la fois rouges et noirs. Les engobes sont d'épaisseur variable (entre $17 \mu \mathrm{m}$ et $82 \mu \mathrm{m}$ ), souvent sur un même tesson (fig. 9). D'une manière générale, la couleur des engobes dépend de leur composition et des conditions de cuisson (température, durée, environnement oxydant ou réducteur). Or, les engobes rouges et les engobes noirs présentent la même composition élémentaire (fig. 10). Comme, au cours de la cuisson, les engobes ont nécessairement atteint les mêmes températures que la terre cuite, on peut en déduire que leurs différences de couleur sont dues aux atmosphères, oxydantes ou réductrices, régnant dans le four. Le cas des formes fermées est simple : les amphorisques ont cuit dans une atmosphère réductrice, d'où leur couleur de surface noire. Pour les formes ouvertes, cuites dans une atmosphère oxydante suivie d'une phase d'enfumage, les potiers ont joué sur les modes d'empilement des vases dans le four. Lorsque la face du bol était directement exposée à l'enfumage, elle devenait noire. Si elle était protégée par un autre vase, elle restait rouge. Une même fournée permettait donc d'obtenir aussi bien des vases Bichrome Red que leurs versions « noircies".

La microspectrométrie Raman offre une confirmation de cette hypothèse. En effet, l'engobe noir est composé exclusivement de magnétite et l'engobe rouge principalement d'hématite, qui sont toutes deux le produit du même composé de fer soumis à des atmosphères de cuisson respectivement réductrice et oxydante (fig. 11). D'ailleurs, à l'intérieur

21. J. Molera, T. Pradell, M. Vendrell-SaZ, "The Colours of Ca-Rich Ceramic Pastes : Origin and Characterization ", Applied Clay Science 13 (1998), p. 187-202. 
de certains engobes noirs, des phases rouges (hématite) ont été observées (fig. 12). En l'occurrence, la phase de cuisson réductrice qui suit la cuisson oxydante n’a pas été assez longue pour que la réaction de transformation d'hématite en magnétite soit complète dans toute l'épaisseur de l'engobe.

\section{LE «BICHROME AMATHOUSIEN »}

La fabrique Bichrome est attestée dans le répertoire chypriote dès le Chypro-Géométrique I mais c'est au Chypro-Archaïque II, période de vogue des décors bariolés et exubérants, qu'elle connaît son plus grand succès. Les deux couleurs qui la caractérisent sont le noir et le rouge, parfois complétés de jaune, de vert ou de bleu (variante Polychrome White 22 .

On a depuis longtemps remarqué l'existence, à Amathonte, d'un décor Bichrome de type particulier, constitué non du contraste entre le noir et le rouge, mais de deux teintes voisines, noir-gris et noir-brun ${ }^{23}$. Son emploi sur des amphorisques du "style d'Amathonte ", de réalisation particulièrement soignée (fig. 13), montre que cette technique est bien voulue et non le résultat d'un accident. Elle est, par ailleurs, attestée beaucoup plus largement dans la production amathousienne. Parmi les séries fabriquées à Amathonte au Chypro-Archaïque II, on mentionnera, outre les amphorisques, les bols sans anses, à parois évasées et lèvre étalée (fig. 14). Enfin, loin de n’apparaître qu’à la fin de la période archaïque, cette technique de décor est connue dès le Chypro-Archaïque I, comme le montrent une cruche à oiseaux conservée au musée du Louvre (fig. 15) et plusieurs amphores de grande taille provenant de la grotte du sanctuaire d'Aphrodite (fig. 2-3 et 16).

Parfois non reconnue - les vases sont alors dits White Painted V24 -, la fabrique est généralement interprétée comme du " pseudo-Bichrome ", c'est-à-dire non pas obtenue à l'aide de deux pigments différents, mais grâce à un même pigment plus ou moins dilué25. Or, l'étude scientifique de cette technique de décor livre de tout autres conclusions.

22. SCE IV/2, p. 68.

23. Karageorghis, des Gagniers 1974, p. 91-93.

24. C'est le cas, par exemple, de la cruche à décor de têtes hathoriques provenant de la tombe 194 d'Amathonte ( $B C H 105$ [1981], p. 1009, fig. 80). Il est vrai que les deux couleurs sont souvent difficiles à distinguer.

25. Karageorghis, des Gagniers 1974, p. 91. 
Seul un tesson de fabrique "pseudo-Bichrome " a été analysé (fig. 17)26. Il s'agit d'un fragment de col d'amphore de grande taille à anses verticales fixées sur le col27, provenant du dépôt de la grotte du sanctuaire (contexte daté du Chypro-Archaïque II). Le décor paraît constitué de deux noirs différents : l'un de teinte plutôt grise, l'autre plus brun. Les données chromamétriques permettent de distinguer ces deux teintes, mais elles ne permettent pas de décider si elles ont été obtenues à partir d'une même matière colorante, plus ou moins diluée, ou à partir de deux pigments différents.

Les analyses élémentaires par spectrométrie X couplée au microscope électronique à balayage (MEB/EDS) montrent que la peinture brune a été réalisée à l'aide d'un oxyde de manganèse (Mn), ce qui a été confirmé par l'analyse en spectrométrie Raman (fig. 18). La bande principale du spectre correspond à celle d'un oxyde de manganèse (psilomélane) régulièrement rencontré sur les décors noirs à base de manganèse des céramiques amathousiennes et compatible avec les ressources géologiques à proximité du site ${ }^{28}$.

Dans la peinture gris-noir, le Mn est absent et l'élément chromogène est le fer (fig. 19). Les matières colorantes utilisées pour le décor des vases "pseudo-Bichrome » sont donc les mêmes que celles utilisées pour le décor des vases Bichrome: le gris-noir correspond au rouge, le brun-noir au noir. Seul l'état d'oxydation du fer, qui résulte de l'atmosphère de cuisson (oxydante ou réductrice), différencie les deux fabriques. Cet élément apparaît sous forme d'hématite $\left(\mathrm{Fe}_{2} \mathrm{O}_{3}\right.$, rouge) dans des conditions de cuisson oxydantes et sous forme de magnétite $\left(\mathrm{Fe}_{3} \mathrm{O}_{4}\right.$, noire) dans des conditions réductrices. Les potiers amathousiens ont joué sur les conditions de cuisson pour obtenir tantôt du rouge, tantôt du noir. Le «Bichrome amathousien " n'est pas issu de l'emploi d'une même matière colorante, plus ou moins délayée : c'est un vrai Bichrome, cuit dans une atmosphère réductrice. Là encore, le jeu sur les cuissons permet aux potiers d'élargir leur répertoire, sans pour autant rompre avec les techniques de décoration traditionnelles.

26. L'autorisation d'exportation, pour la durée d'un an, spécifiait que l'échantillon ne devait pas être altéré. Toutes les analyses ont donc été effectuées selon des méthodes non destructives (MEB/EDS, Spectrométrie Raman) sur le tesson entier, qui a été restitué intact au terme de l'étude.

27. Du même type que les amphores illustrées fig. 2-3 et $\mathbf{1 6 .}$

28. Th. M. PAntazis, "The Geology and Mineral Resources of the Pharmakas-Kalavasos Area ", Geological Survey Department of Cyprus, Memoir 8 (1967), p. 35. 


\section{L'IMITATION DE LA TECHNIQUE DE LA FIGURE NOIRE}

Dans la deuxième partie du Chypro-Archaïque II (à partir de ca 550 av. J.-C.), les ateliers amathousiens créent un style figuré, combinant différentes influences thématiques, égyptienne, phénicienne et grecque. Ce «style d'Amathonte» est le plus souvent employé pour décorer de petits amphorisques, dont les traits morphologiques sont caractéristiques des tendances locales (fig. 20), plus rarement des cruches à embouchure trilobée (fig. 21), exceptionnellement une cruche à bec ${ }^{29}$. Les vases sont le plus souvent ornés d'un décor Bichrome, fréquemment, on l'a vu, en "Bichrome amathousien ».

De qualité d'exécution variée, les vases du «style d'Amathonte » ont un répertoire décoratif relativement restreint : entièrement peints, ils portent des frises de motifs géométriques ou floraux sur le col et le bas de la panse, ainsi qu'un décor organisé en métopes sur la zone de plus grand diamètre, entre les anses ${ }^{30}$. Le panneau central est occupé par une tête hathorique (fig. 22) ou d'autres images associées à la personnalité et au culte de la grande déesse amathousienne : végétaux - réalistes ou stylisés (fig. 13 et 20) -, animaux - réels ou monstrueux -, différents objets cultuels ${ }^{31}$. Plus rarement,

29. Cruche à bec: Amathonte T. 194/18 (BCH 105 [1981], p. 1009, fig. 80); cruches à embouchure trilobée : Limassol T. 177 (BCH 106 [1982], p. 704, fig. 57), Louvre inv. AM 197 (CVA France 5, Louvre 4, pl. 8, 8 et 10), Louvre inv. AM 393A (KarageOrghis, des Gagniers 1974, p. 511), Newcastle-upon-Tyne, University Museum inv. 119 (KARAGEORGHIS 1989, pl. 16, 1-3).

30. Pour l'étude thématique des décors, cf. A. Hermary, "Le "style d'Amathonte" ", in KarageOrghis, LAFFINEUR, VANDENABEELE 1997, p. 157-161, pl. LI-LII.

31. Tête hathorique : Amathonte T. 129 (Karageorghis, des Gagniers 1974, p. 107, no 4 et p. 506, no 3), Amathonte T. 192/58.1 (BCH 107 [1983], p. 913, fig. 21), Amathonte T. 194/18 (BCH 105 [1981], p. 1009, fig. 80), Amathonte T. NW 194-I (BCH 102 [1978], p. 957, fig. 35), Amathonte T. 747/78 (BCH 126 [2002], p. 702, fig. 46), Amathonte-Terrasse Ouest (A. Hermary (supra), pl. LII, b; HermarY 1986, pl. XXXV, 2a et b), Amathonte-dépôt du rempart Nord (BCH117 [1993], p. 715, fig. 67 = fig. 22), Idalion (Karageorghis, Des Gagniers 1974, p. 509, nº 6), Myrina ou Phocée (Karageorghis, des GAGNIERS 1974, p. 505, no 2), provenance inconnue (Collection Piéridès = KaragEOrghIS, des Gagniers 1974, p. 504, no 1 ; Nicosie inv. B 388 = ibid., p. 508, no 5 ; Louvre inv. AM 393A = ibid., p. 511). Motifs végétaux : Amathonte T. 37 (CVA Great Britain 2, British Museum 2, pl. 12, 4 et 9), Amathonte T. 72 (ibid., pl. 12, 10), Amathonte T. 84 (ibid., pl. 12, 8), Amathonte T. 106 (ibid., pl. 12, 6, 1, 2 et 5), Amathonte T. 204/27 (BCH 105 [1981], p. 1010, fig. 86), Amathonte T. 204/30 (ibid., p. 1010, fig. 85), Amathonte T. 342/5 et 10 (BCH 106 [1982], p. 699, fig. 40-41), Amathonte T. 440 (BCH 111 [1987], p. 697, fig. 54), Amathonte T. 470/43 (BCH 103 [1979], p. 680, fig. 20), Amathonte T. 480/1 (KarageOrghis 1989, pl. 17, 4), Amathonte T. 572/6 (BCH 114 [1990], p. 950, fig. 46), AmathonteKavkalia (BCH 117 [1993], p. 726, fig. 22), Limassol-Agios Spyridon T. 196 (BCH 111 [1987], p. 724, fig. 204), Limassol-Agios Athanasios T. 581/1 (BCH 101 [1977], p. 724, fig. 39), "Amathonte" (British Museum inv. C 964 = CVA Great Britain 2, British Museum 2, pl. 12, 12 ; Genève inv. P 242 = V. KARAGeOrghis, Ancient Cypriote Art in the Musée d'Art et d'Histoire, Geneva [2004], no 118), KalavassosKafkallies T. 3 (BCH 108 [1984], p. 925, fig. 111), de provenance inconnue (Bonn, Akademisches 
l'organisation en panneaux cède la place à de véritables scènes figurées ${ }^{32}$.

Tous les commentateurs s'accordent à reconnaître, dans cette série locale, l'influence de la peinture de vases grecque contemporaine ${ }^{33}$. De fait, même si beaucoup de motifs trouvent leur source dans le répertoire proche-oriental, leur rendu stylistique trahit un modèle grec. C'est le cas, par exemple, d'un amphorisque fragmentaire découvert dans le dépôt archaïque du rempart Nord (fig. 23) : le motif végétal central (fleurs de lotus ouvertes et en bouton sur un monticule) est d'origine égyptienne, le schéma général (deux animaux de part et d'autre d'un arbre stylisé) est plus largement oriental, mais les deux chèvres, dont on devine les traits en silhouette noire, semblent échappées d'un vase du "Wild Goat Style ». Cette impression est d'ailleurs renforcée par le recours à des incisions qui, recoupant le tracé en silhouette noire ou rouge (technique chypriote Bichrome), imitent le procédé de la figure noire (fig. 24). Les incisions, parfois limitées à quelques détails, sont fréquentes sur les vases les plus soignés de la série (fleurs de lotus et chevelure de la tête hathorique sur l'amphorisque de la fig. 22, par exemple).

Un autre trait technique, propre au «style d'Amathonte " est la qualité de la pâte utilisée : elle est, contrairement au tout-venant de la production amathousienne, très fine et très blanche. Or, parmi les influences, celle des styles de la Grèce de l'Est, et notamment

Kunstmuseum inv. 761 = KARAGEORGHIS, des GAGNIERS 1974, p. 514, no 11 ; Dublin inv. NMI 1940:22 $=$ Chr. Souzoudzoglou-HaYwood, Cypriot Antiquities in Dublin [2004], no 141 ; Louvre inv. AM $188=$ CVA France 5, Louvre 4, pl. 7, 1 et 3; San Francisco Bay Area Collections = V. KARAGEORGHIS, D. A. AMYX et al., Corpus of Cypriote Antiquities 5, SIMA XX/5 [1974], n ${ }^{\circ} 67$; Sarajevo cat. $\mathrm{n}^{\circ} 24=$ CVA Yougoslavie 4, pl. 5, fig. 2 et 5). Animaux : poisson (Amathonte T. 52 = KARAGEORGHIS, des GAGNIERs 1974, p. 515, $\mathrm{n}^{\mathrm{o}} 12$ ) ; oiseau, en particulier coq (Amathonte T. 470/43 = KARAGEORGHIS 1989, pl. 17, 3 ; de provenance inconnue : Nicosie inv. B 22 = KARAGEORGHIS, des GAGNIERS 1974, p. 513, no 10 ; Nicosie inv. 1952/XII27/1 = ibid., p. 512, no 9). Sphinx : Amathonte-Terrasse Ouest (HERMARY 1986, pl. XXXV, 1). Aryballe : de provenance inconnue (Varsovie, Musée National inv. 205 = CVA Pologne 1, pl. 4, fig. 6a-c).

32. Scène de banquet en plein air : Amathonte T. 129 (KARAgEOrghis, des GaGNiERS 1974, p. 516-517, $\mathrm{n}^{\mathrm{o}}$ 13) ; cavalier et personnage à pied : Amathonte T. 251/8 (BCH 105 [1981], p. 1012, fig. 95) ; différentes scènes fragmentaires avec personnages : Amathonte-sanctuaire d'Aphrodite (HERMARY 1986, pl. XXXV, 3-7) ; oiseaux ou sphinx affrontés de part et d'autre d'un motif végétal stylisé : Limassol, T. 166 (BCH 106 [1982], p. 704, fig. 57), de provenance inconnue (Princeton, University Art Museum = V. KARAGEORGHIS, RDAC 1990, pl. XXII ; Newcastle-upon-Tyne, University Museum inv. 199 = KARAGEORGHIS 1989, pl. 16, 1-3); scène de sacrifice devant un chapiteau hathorique (Louvre inv. AM 393D = KARAGEORGHIS, des GAGNIERS 1974, p. 510, nº 7, de provenance inconnue).

33. Cf. notamment KarageOrghis, des Gagniers 1974, p. 91-93 et KaragEORGHIS 1989. Plus récemment: St. BÖHM, «A Flower-Smelling Man. About a Pictorial Motif of Cypro-Archaic Vase-Painting, Its Iconography and Significance ", in V. KarageOrghis, H. MatTHÄUS, S. Rogge (éds), Cyprus : Religion and Society from the Late Bronze Age to the End of the Archaic Period (2005), p. 91-98. 
celle de la céramique chiote, est prégnante ${ }^{34}$. Sans prétendre à l'exhaustivité, on pourra mentionner les frises décorant le bas de la panse (fleurs de lotus [fig. 22] ${ }^{35}$ ou triangles [fig. 23] ${ }^{36}$ ), certaines stylisations des fleurs de lotus (fig. 20) ${ }^{37}$, les représentations d'oiseaux (fig. 21) ${ }^{38}$ et de coqs ${ }^{39}$, et même certaines scènes figurées ${ }^{40}$. Il est d'ailleurs remarquable que, seule avec les céramiques du style de Fikellura, de Clazomènes et les hydries de Caéré, la céramique chiote représente parfois cet ornement si typiquement chypriote que sont les " couvre-oreilles ${ }^{41}$ ". Si donc il ne fait aucun doute que l'aspect de surface des vases chypriotes du «style d'Amathonte " imite l'épais engobe blanc qui orne les productions chiotes, encore faut-il s'assurer qu'il s'agit du même procédé technique. Ce dernier est, en effet, étranger à la tradition amathousienne qui répugne généralement à recouvrir d'engobe les vases de fabrique White Painted ou Bichrome.

Les échantillons d'amphorisques du "style d'Amathonte " analysés proviennent du dépôt du rempart Nord (contexte daté du Chypro-Archaïque II). Leur surface est lisse et brillante (abstraction faite des concrétions calcaires). Ils présentent le décor caractéristique de la fabrique sur leur face externe, en Bichrome ou "Bichrome amathousien » sur fond clair. Sur l'un des échantillons, des incisions ont été faites sur le décor peint (fig. 24).

L'épaisseur des fragments varie entre 2 et $7 \mathrm{~cm}$. Leur texture se caractérise par une terre cuite très fine, présentant une faible porosité et des inclusions de dimensions très réduites (de quelques micromètres à $0,1 \mathrm{~mm}$ ). Ces dernières sont peu nombreuses (concentration maximale de $4 \%$ ). Les observations au microscope électronique à balayage montrent que ces céramiques ne comportent pas d'engobe (fig. 25).

34. Cf. St. BöHm (supra, n. 30), à propos du motif du personnage. C'est également ce que note Fr. Vandenabeele pour une série marginale de cruches à choéphore, décorées dans ce qu’elle nomme le « Pseudo Black Figured Style ». Sur les 7 exemplaires de provenance connue, 2 ont été trouvés à Amathonte, 2 à Kourion, 2, voire 3, à Marion : Fr. VANDENABEELE, "The Pictorial Decoration on the Cypriote Jugs with Figurines Holding an Oinochoe ", in Karageorghis, Laffineur, Vandenabeele 1997, p. 130-133. Elle remarque, à juste titre, que cette série entretient d'étroits liens, stylistiques et iconographiques, avec le "style d'Amathonte ", dont elle est, par ailleurs, contemporaine.

35. Cf. A. A. Lemos, Archaic Pottery of Chios. The Decorated Styles (1991), pl. 12, 114 et pl. 13, 124.

36. Ibid., pl. 43, 310 .

37. Ibid., pl. 23, 247.

38. Ibid., pl. 40, 303 et pl. 224.

39. Ibid., pl. 204-206, 208 et 213.

40. On rapprochera, par exemple, le tesson du sanctuaire d'Aphrodite représentant des femmes au milieu de fleurs de lotus (HERMARY 1986, pl. XXXV, 6) d'un fragment de calice provenant d'Égine (A. A. LEMOS [supra, n. 35], pl. 137, 978).

41. J. M. HemelrijK, "Some Ear Ornaments in Archaic Cypriot and East Greek Art », BaBesch 38 (1963), p. $28-51$. 
L'observation en lumière naturelle de coupes épaisses polies révèle qu'elles ont une matrice argileuse beige rosé. Leur texture est fine, le dégraissant est invisible à l'œil nu. Sous bombardement électronique, la couleur des pâtes est variable : rouge (caractéristique des terres cuites calcaires) ou violine (propre aux pâtes siliceuses). Ces différences sont dues à l'état d'altération de certaines des céramiques. En effet, l'examen par cathodoluminescence montre un phénomène, remarqué par ailleurs sur des cruches Black-on-Red: une zone non luminescente délimitée et parallèle à la face interne de la céramique, accompagnée d'une porosité plus importante ${ }^{42}$. Les analyses chimiques élémentaires par spectrométrie $\mathrm{X}$ couplée au microscope électronique à balayage (MEB/EDS) ${ }^{43}$ permettent d'expliquer que ces zones non luminescentes sont dues à la dissolution de la calcite par le contenu de la céramique (par exemple, un liquide acide).

L'échantillon BDX 9278, moins altéré, possède une concentration en calcium (exprimée en pourcentages d'oxyde : $\mathrm{CaO}$ ) très importante (environ $44 \%$, fig. 26), alors que les analyses de terre cuite (hors dégraissant) réalisées sur plus de soixante tessons de différentes fabriques donnent un teneur moyenne en calcium de $19 \%$ avec un écart-type de $5 \%$. Il est possible que les amphorisques du « style d'Amathonte » aient été réalisés avec une marne plus riche en calcium que l'argile utilisée pour les autres séries de production locale. Mais l'hypothèse la plus simple, et donc la plus probable, est que le potier a ajouté à l'argile habituelle de la chaux (sous forme de calcite pilée et calcinée), dans le but d'abaisser la température de fusion et, partant, d'augmenter la dureté et la blancheur de ces céramiques fines et non dégraissées. Les artisans amathousiens auraient donc imité l'aspect de surface des céramiques chiotes, non pas en recouvrant d'un engobe blanc la matrice de terre cuite, mais en transformant la pâte elle-même (décantation et lavage pour épurer la matrice des dégraissants naturels et obtenir une texture fine, ajout de chaux pour obtenir une teinte blanche).

Aussi différentes soient-elles, les variantes amathousiennes des modes de décoration chypriote reposent sur des procédés techniques relativement limités, et notamment sur un jeu parfaitement maîtrisé des cuissons. L'inventivité des potiers est d'autant plus remarquable qu'ils ne s'affranchissent jamais d'un répertoire établi dès l'époque géométrique : les fabriques «Blackened» ne sont que des versions " noircies " de vases généralement réalisés selon des techniques de décor sur engobe rouge; le "Bichrome

42. Fl. Daniel, S. Fourrier, "Programme d'analyses physico-chimiques de la céramique amathousienne archaïque ", BCH 128-129 (2004-2005), p. 1075-1077.

43. Les analyses élémentaires au MEB/EDS montrent que la teneur en $\mathrm{CaO}$ de l'échantillon BDX 9279 est trois fois plus faible (environ $10 \%$ au lieu de $32 \%$ ) dans la zone non luminescente (face interne de la céramique). La teneur en oxydes de fer, en revanche, est constante (environ $6 \%$, exprimés en pourcentages de $\mathrm{Fe}_{2} \mathrm{O}_{3}$ ). La non-luminescence de la zone interne est donc liée à une faible teneur en carbonates. 
amathousien " n'est rien d'autre qu'un Bichrome de tradition chypriote ; quant à l'imitation de la technique de la figure noire, si elle utilise l'incision, elle marie le plus souvent ses silhouettes noires avec des silhouettes rouges, selon le schéma chypriote traditionnel. Ce n'est pas là un fait nouveau : l'étude que J. N. Coldstream a consacrée aux skyphoi géométriques d'origine égéenne a bien montré que ces derniers étaient amplement diffusés dans l'île et qu'ils y ont suscité des imitations, mais qu'aucune d'entre elles n'était fidèle à ses modèles, le trait le plus frappant étant le recours à une technique de décoration Bichrome ${ }^{44}$.

De fait, si elle est généreusement ouverte aux influences étrangères, la céramique chypriote archaïque reste profondément attachée aux traditions techniques géométriques. C'est ce qui fait l'efficacité du système de classification générale, établi par E. Gjerstad, et c'est le paradoxe de l'artisanat chypriote archaïque. Cette fidélité aux techniques locales suscite des créations que l'on peut juger virtuoses, ou d'une complication absurde. C'est le cas, par exemple, d'une amphore du "style d'Amathonte », le plus grand exemplaire connu à ce jour (fig. 27). Une coupe à travers les couches successives de décor a montré que la céramique était entièrement engobée de rouge, cette première surface étant ensuite recouverte de peinture blanche, support du décor figuré réalisé en noir et rouge. Selon la terminologie suédoise, il s'agit donc d'une amphore de fabrique Bichrome Red II(V), alors que l'effet obtenu est celui d'un vase Bichrome à surface très blanche. Contrairement aux autres vases de la série, des amphorisques de taille réduite, les dimensions importantes de l'amphore nécessitaient d'ajouter beaucoup de dégraissant, afin de servir d'armature lors des tensions suscitées par l'évaporation de l'eau au moment du séchage et de la cuisson. Il était donc indispensable de recouvrir le vase d'une épaisse couverte qui dissimulât les imperfections de la pâte. L'engobe blanc étant étranger aux traditions chypriotes, le peintre a décidé d'utiliser la technique traditionnelle des fabriques Bichrome Red, mais en transformant la peinture blanche, habituellement réservée à des motifs décoratifs limités, en deuxième couche de surface, comme s'il s'agissait d'un deuxième engobe. Ce vase est, à plus d'un titre, exceptionnel, mais il s'inscrit parfaitement dans les techniques traditionnelles de fabrication.

L'étude des procédés décoratifs ne constitue qu'un des aspects du programme de recherche portant sur la céramique amathousienne archaïque. Les résultats obtenus montrent l'intérêt des analyses scientifiques, hors des datations et des provenances, domaines où on les cantonne bien trop souvent. En l'absence de découverte des vestiges matériels d'ateliers, et notamment de restes de fours, elles permettent d'appréhender, dans une certaine mesure, les pratiques d'un artisanat.

44. J. N. Coldstream, "Geometric Skyphoi in Cyprus », RDAC 1979, p. 255-269, pl. XXIX-XXXI. 


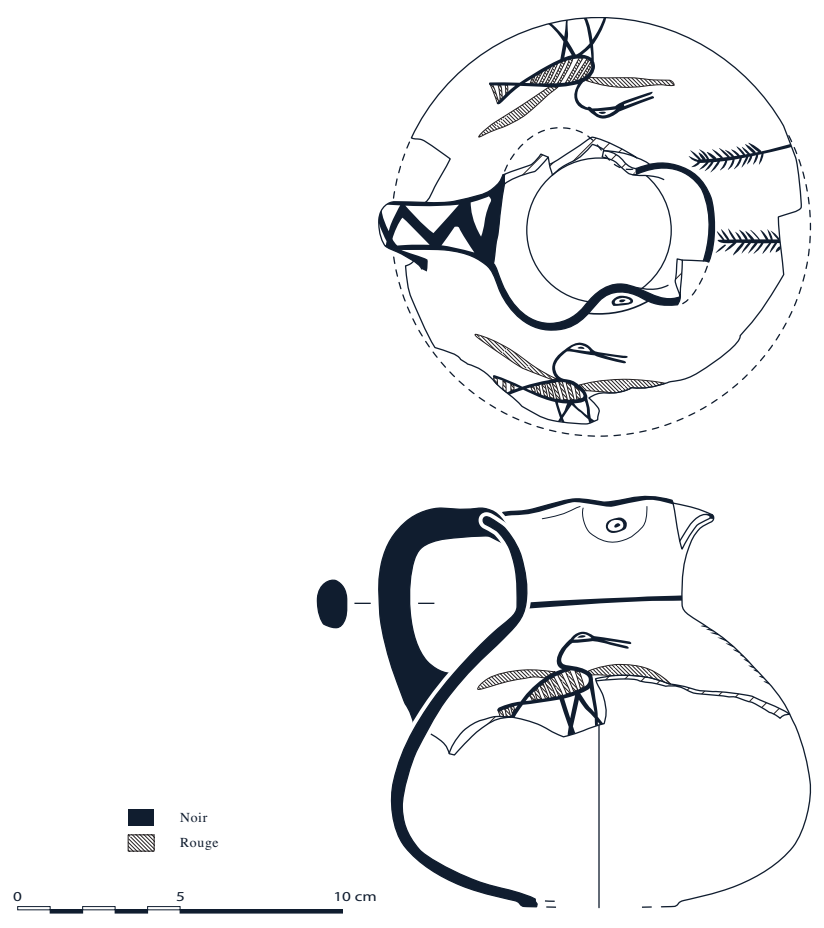

Fig. 1. - Cruche Bichrome IV provenant du sanctuaire d'Aphrodite (dessin EFA, J. Humbert). 


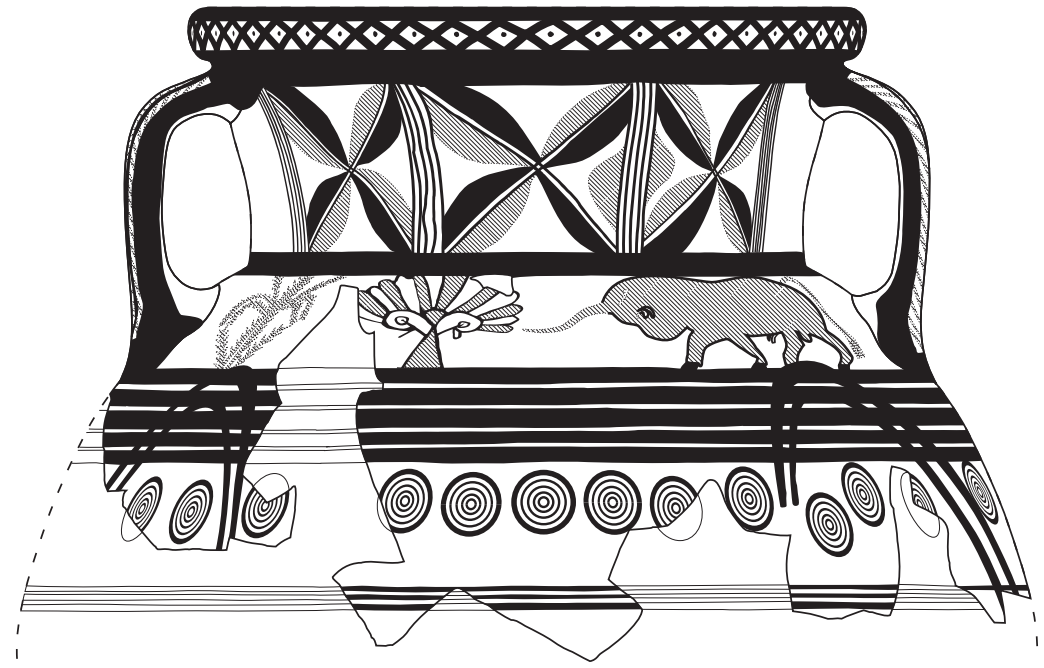

Fig. 2. - Amphore aux taureaux de la grotte du sanctuaire. Musée de Limassol, AM 1554 (dessin EFA, J. Humbert). 


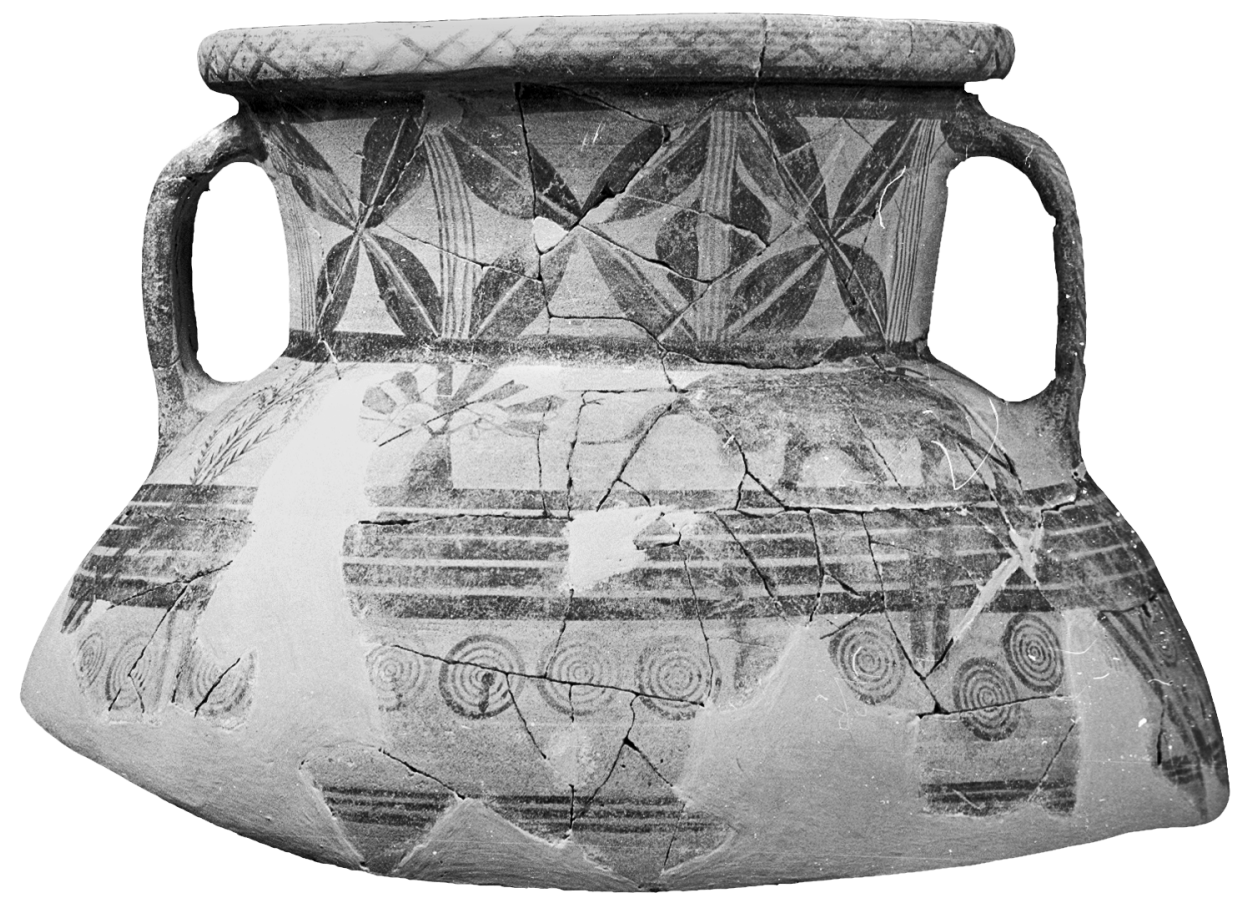

Fig. 3. - Id. (cliché EFA, Ph. Collet). 


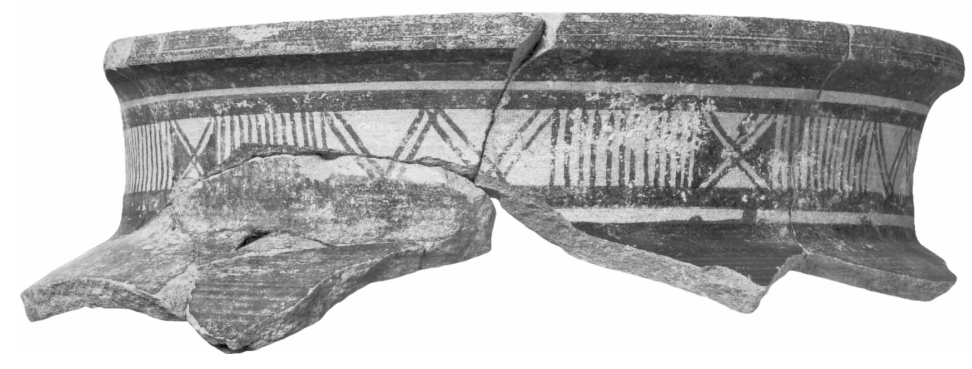

Fig. 4. - Col d'amphore de style hybride provenant du sanctuaire d'Aphrodite. Le col est décoré en noir sur surface claire (White Painted), le reste du vase en noir sur engobe rouge (Black-on-Red) (cliché EFA, Ph. Collet).

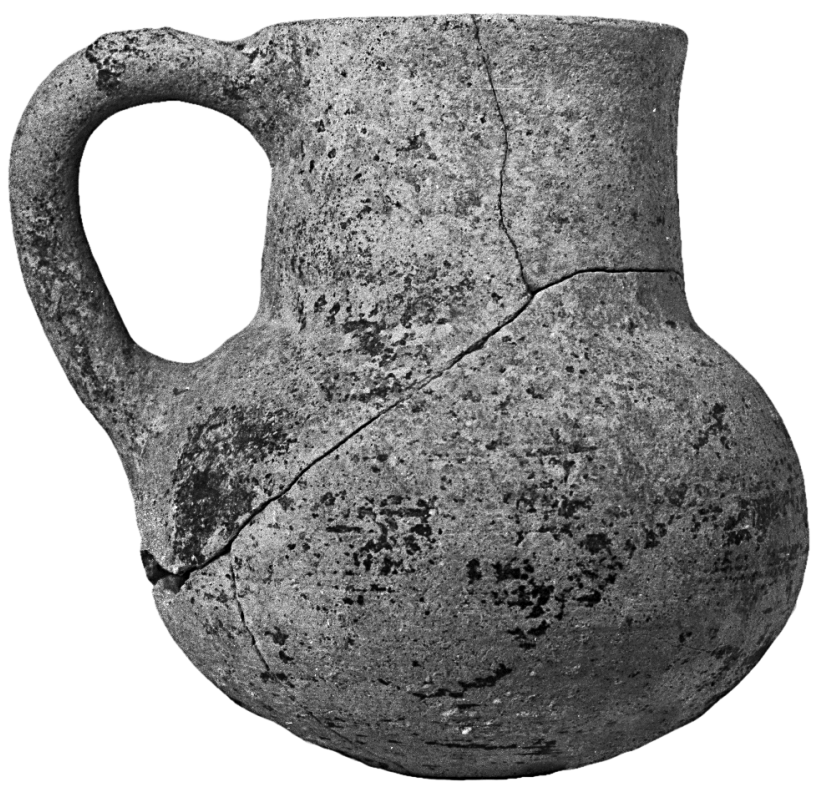

Fig. 5. - Cruche Black Slip V provenant de la grotte du sanctuaire (cliché EFA, Ph. Collet). 
Peinture noire

Peinture blanche

Engobe rouge
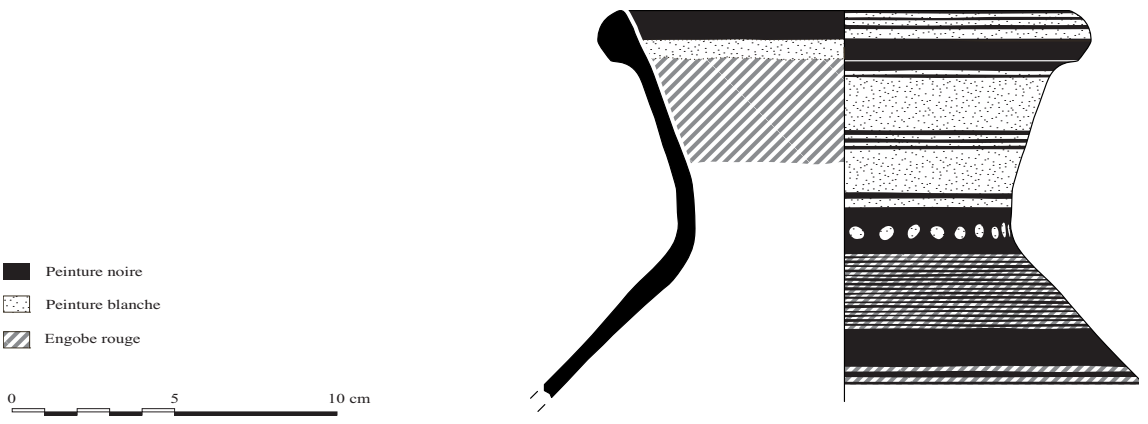

Fig. 6. - Amphore Bichrome Red II(V) provenant du dépôt du rempart Nord (dessin EFA, J. Humbert).
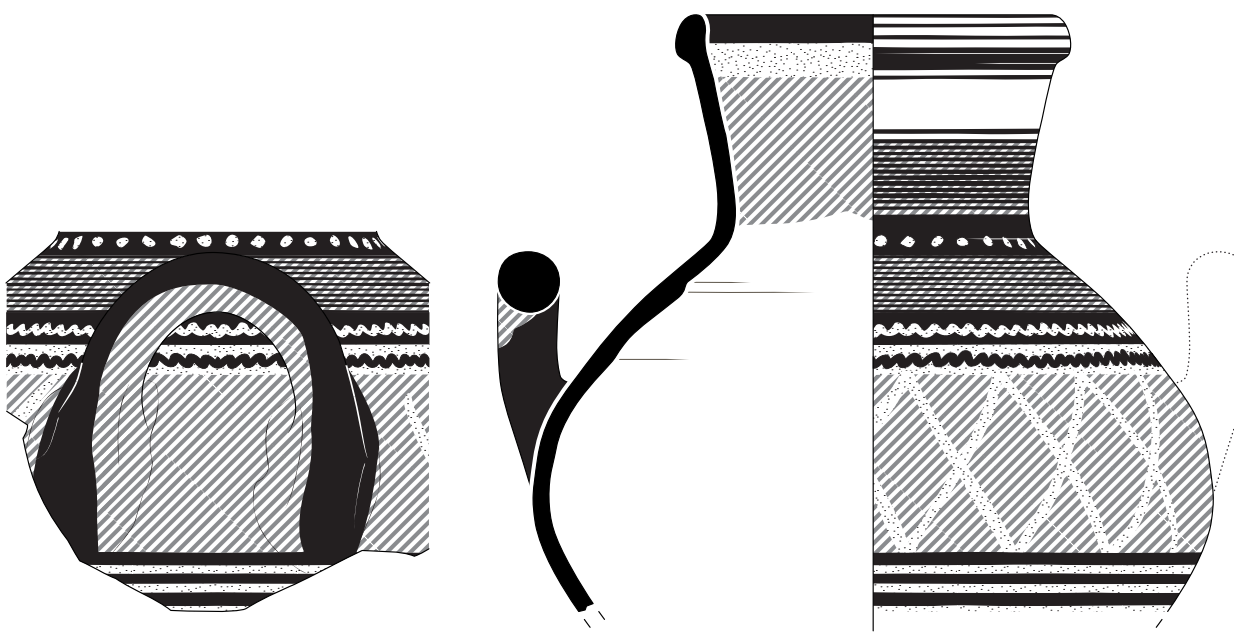

Peinture noire

Peinture blanche

Engobe rouge, viré au noir

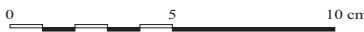

Fig. 7. - Amphore Bichrome Red II(V) «Blackened » de même provenance (dessin EFA, J. Humbert). 


\begin{tabular}{|c|c|c|c|c|c|c|}
\hline 崌 & 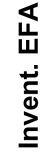 & 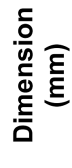 & 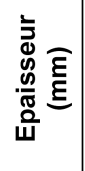 & 응 & Décor & Type céramique \\
\hline ఫ్ & 尔 & 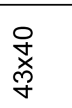 & 5 & 10,4 & une bande noire sur fond noir & $\begin{array}{l}\text { Amphorisques bichr. } \\
\text { Red « blackened » }\end{array}$ \\
\hline ஜ̊ & $\stackrel{\substack{+\dot{\infty}}}{\infty}$ & $\begin{array}{l}\text { 乃े } \\
\underset{x}{\infty} \\
0 \\
0\end{array}$ & 5 & 14,2 & $\begin{array}{l}\text { Alternance bandes incurvées noires et } \\
\text { blanches sur fond noir }\end{array}$ & 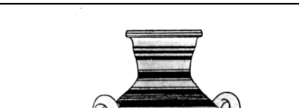 \\
\hline ஜ্ঠি & 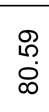 & 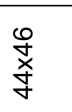 & 7 & 16,5 & une bande noire et une blanche sur fond noir & 15 \\
\hline 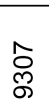 & $\begin{array}{l}\text { ¿ै } \\
\dot{\infty}\end{array}$ & ヘิ & 8 & 7,6 & $\begin{array}{l}\text { Ext. fond rouge, } \\
\text { Int. fond noir }\end{array}$ & $\begin{array}{l}\text { Bols bichr. Red ou BoR } \\
\text { "blackened". }\end{array}$ \\
\hline $\begin{array}{l}\infty \\
\check{\varnothing}\end{array}$ & @i & $\begin{array}{l}\stackrel{2}{N} \\
\underset{x}{x} \\
\text { ల }\end{array}$ & 6 & 6 & $\begin{array}{l}\text { Ext. fond rouge } \\
\text { Int. une bande noire sur fond noir }\end{array}$ & \\
\hline ஓ্ণ & 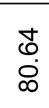 & $\begin{array}{l}\stackrel{\infty}{\sim} \\
\underset{x}{\sim} \\
\stackrel{f}{\sigma}\end{array}$ & 4 & 5,8 & $\begin{array}{l}\text { Ext. fond rouge } \\
\text { Int. bandes noires (et blanches ?) sur fond noir }\end{array}$ & \\
\hline$\frac{}{\grave{m}}$ & $\begin{array}{l}\infty \\
\stackrel{0}{0} \\
\infty\end{array}$ & $\begin{array}{c}\bar{x} \\
\text { ले }\end{array}$ & 4 & 2,2 & $\begin{array}{l}\text { Ext. lignes noires (et blanches ?) sur fond noir } \\
\text { Int. deux bandes noires sur fond rouge }\end{array}$ & $\begin{array}{l}\text { Bols bichr. Red ou BoR } \\
\text { "blackened". }\end{array}$ \\
\hline$\underset{\delta}{\sigma}$ & $\begin{array}{l}8 \\
: \\
\infty\end{array}$ & $\begin{array}{l}\mathscr{P} \\
\underset{x}{\infty} \\
\text { o }\end{array}$ & 4 & 5,5 & $\begin{array}{l}\text { Ext. une bande noire sur fond noir-brun } \\
\text { Int. deux bandes noires sur fond rouge }\end{array}$ & \\
\hline$\stackrel{N}{\check{m}}$ & $\begin{array}{l}\overline{1} \\
\dot{\infty}\end{array}$ & $\begin{array}{l}\text { ָ̃ } \\
\text { ơ }\end{array}$ & 8 & 4,3 & $\begin{array}{l}\text { Ext. fond noir } \\
\text { Int. une bande noire sur fond rouge }\end{array}$ & \\
\hline
\end{tabular}

Fig. 8. - Échantillons provenant d'amphorisques et de bols Bichrome Red "Blackened (CRPAA). 


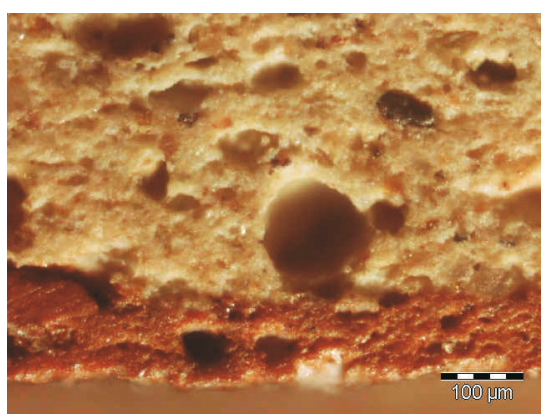

Fig. 9. - Image au microscope optique, en lumière naturelle, d'un engobe (BDX 9311), agrandissement x 200 (cliché CRPAA).

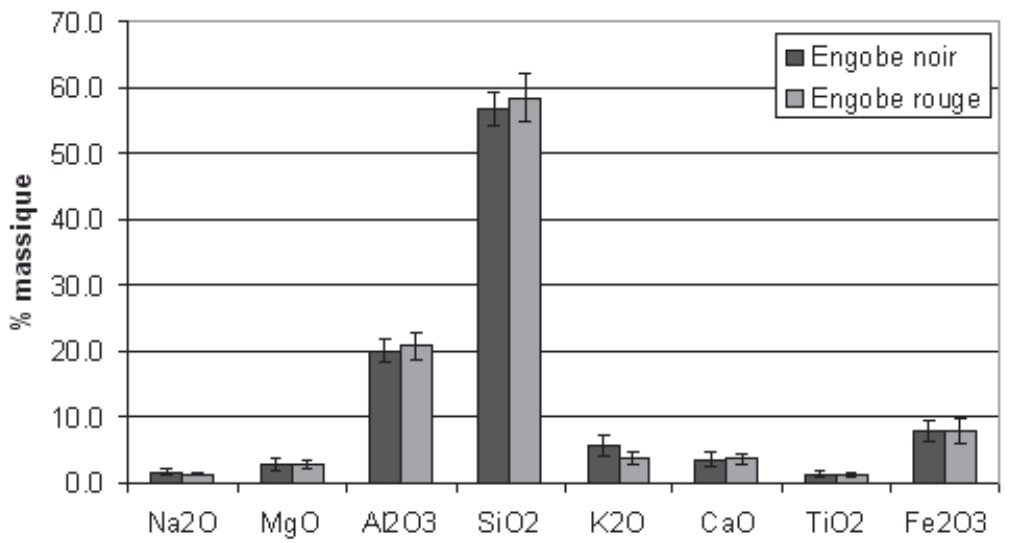

Fig. 10. - Comparaison de la composition élémentaire moyenne (analyse EDXS), exprimée en pourcentages pondéraux d'oxydes, des engobes noirs et rouges des céramiques analysées (Cf. tableau fig. 8). 


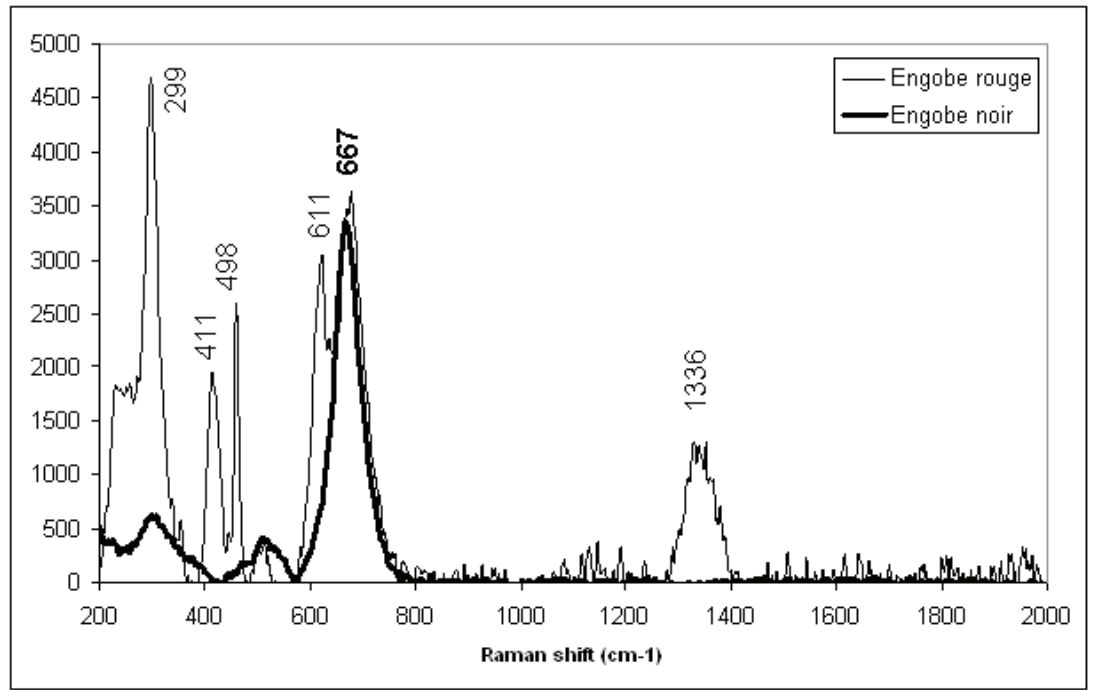

Fig. 11. - Spectres Raman de l'engobe noir de la face interne (magnétite $\left[\mathrm{Fe}_{3} \mathrm{O}_{4}\right]$, en gras) et rouge de la face externe (hématite $\left[\mathrm{Fe}_{2} \mathrm{O}_{3}\right]$ + magnétite, en maigre) d'un même bol "Blackened " (BDX 9309). Lors de la dernière phase de cuisson oxydante, tous les oxydes de fer de la face externe ne s'oxydent pas en hématite et les deux espèces (magnétite et hématite) sont présentes. En revanche, sur la face interne, protégée de l'oxydation, l'engobe noir ne contient que de la magnétite comme l'indique sa bande caractéristique à $667 \mathrm{~cm}^{-1}$ (CRPAA).

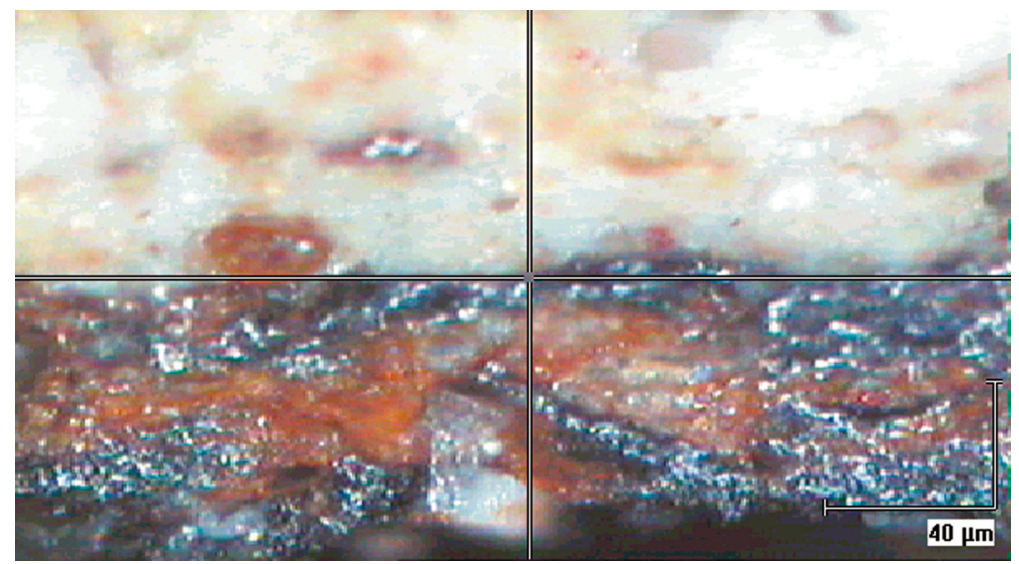

Fig. 12. - Image au microscope optique, en lumière naturelle, d'un engobe (BDX 9307) (cliché CRPAA). 

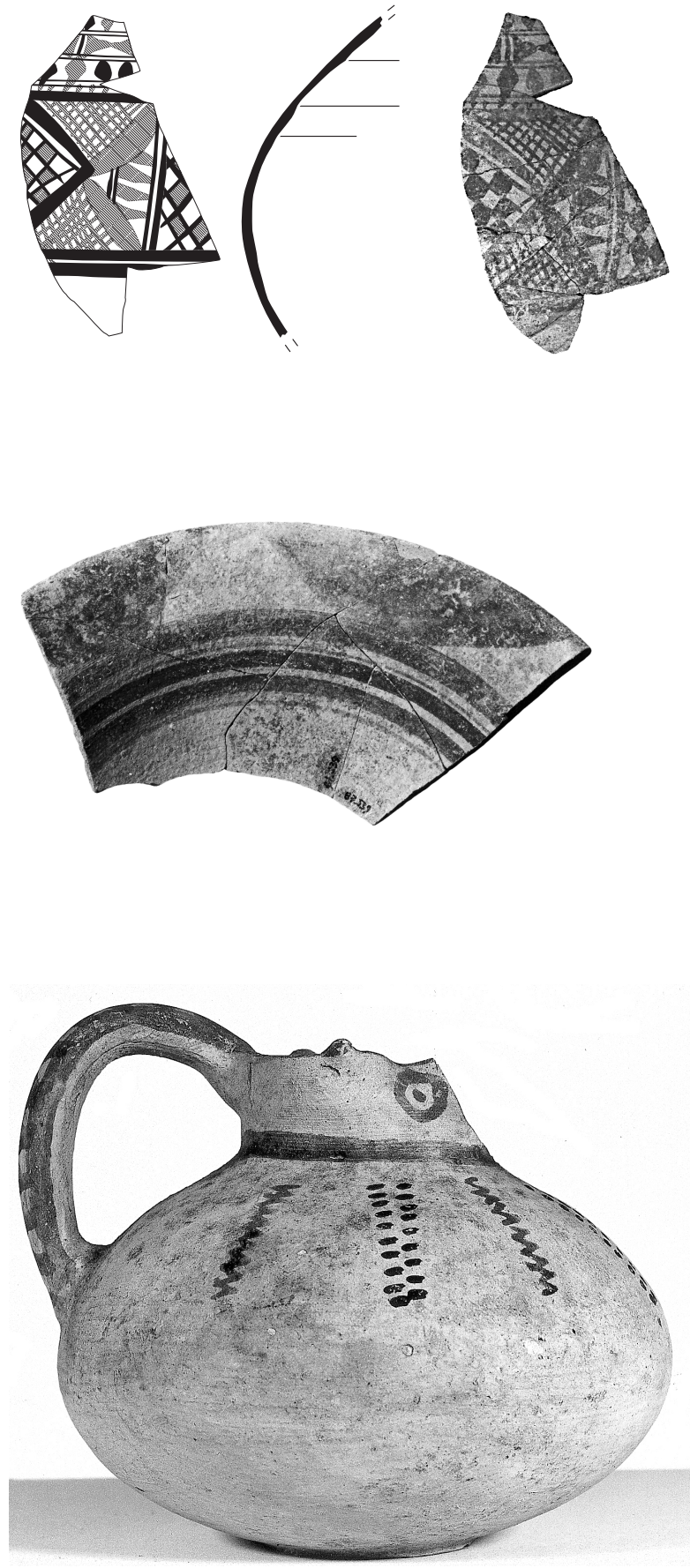

Fig. 13. - Fragment de panse d'un amphorisque du «style d'Amathonte " à décor en "Bichrome amathousien " (dessin EFA, J. Humbert; cliché EFA, Ph. Collet).

Fig. 14. - Bol à lèvre étalée et décor en "Bichrome amathousien " provenant de la grotte du sanctuaire (cliché EFA, Ph. Collet).

Fig. 15. - Cruche Bichrome IV à décor en "Bichrome amathousien". Musée du Louvre, inv. MNB 71 (cliché du musée). 


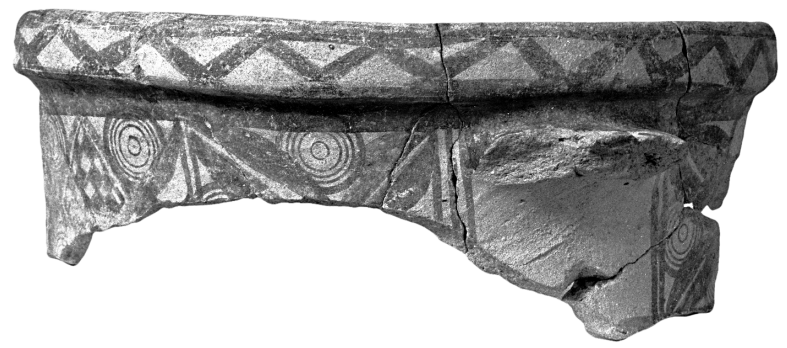

Fig. 16. - Amphore de grande taille à décor en "Bichrome amathousien ", provenant de la grotte du sanctuaire (cliché EFA, Ph. Collet).

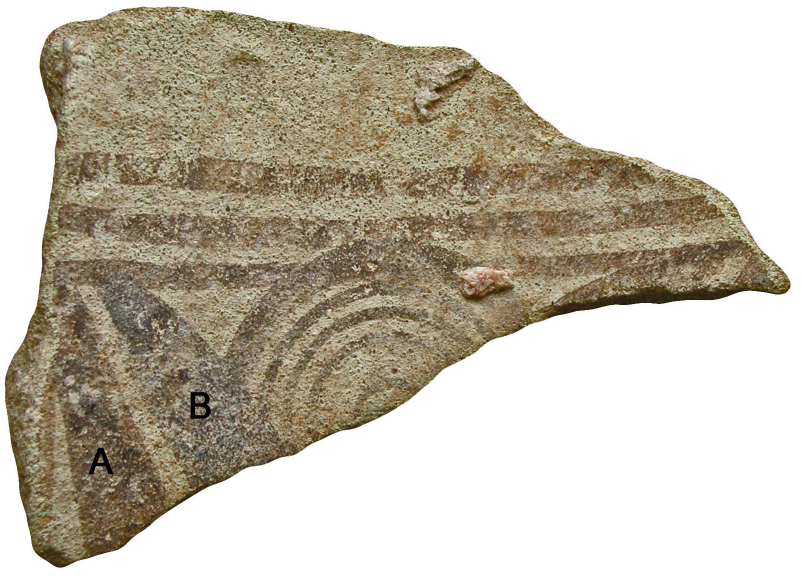

Fig. 17. - Échantillon à décor "pseudo-Bichrome" (BDX 9301). Deux types de noirs composent le décor, l'un à dominante brune (A), l'autre plus gris (B) (cliché CRPAA). 


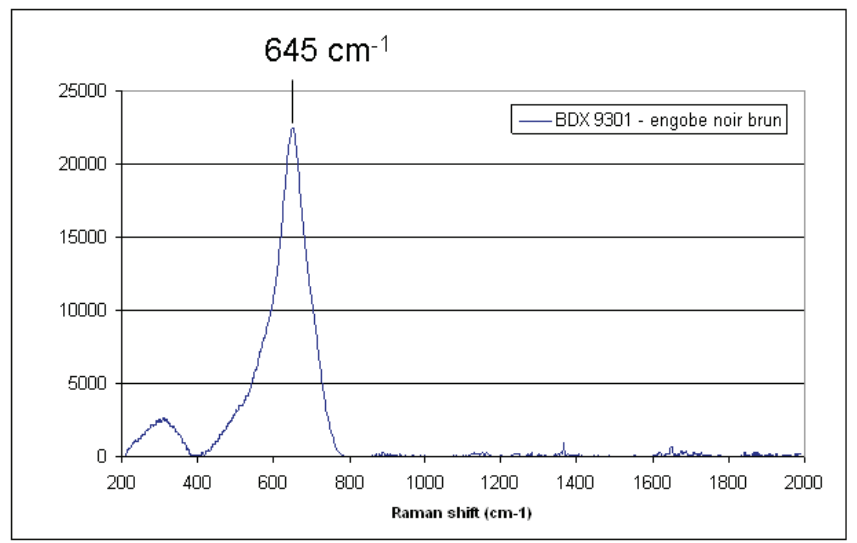

Fig. 18. - Spectre Raman du décor brun (céramique à décor " pseudo-Bichrome », BDX 9301). (CRPAA).
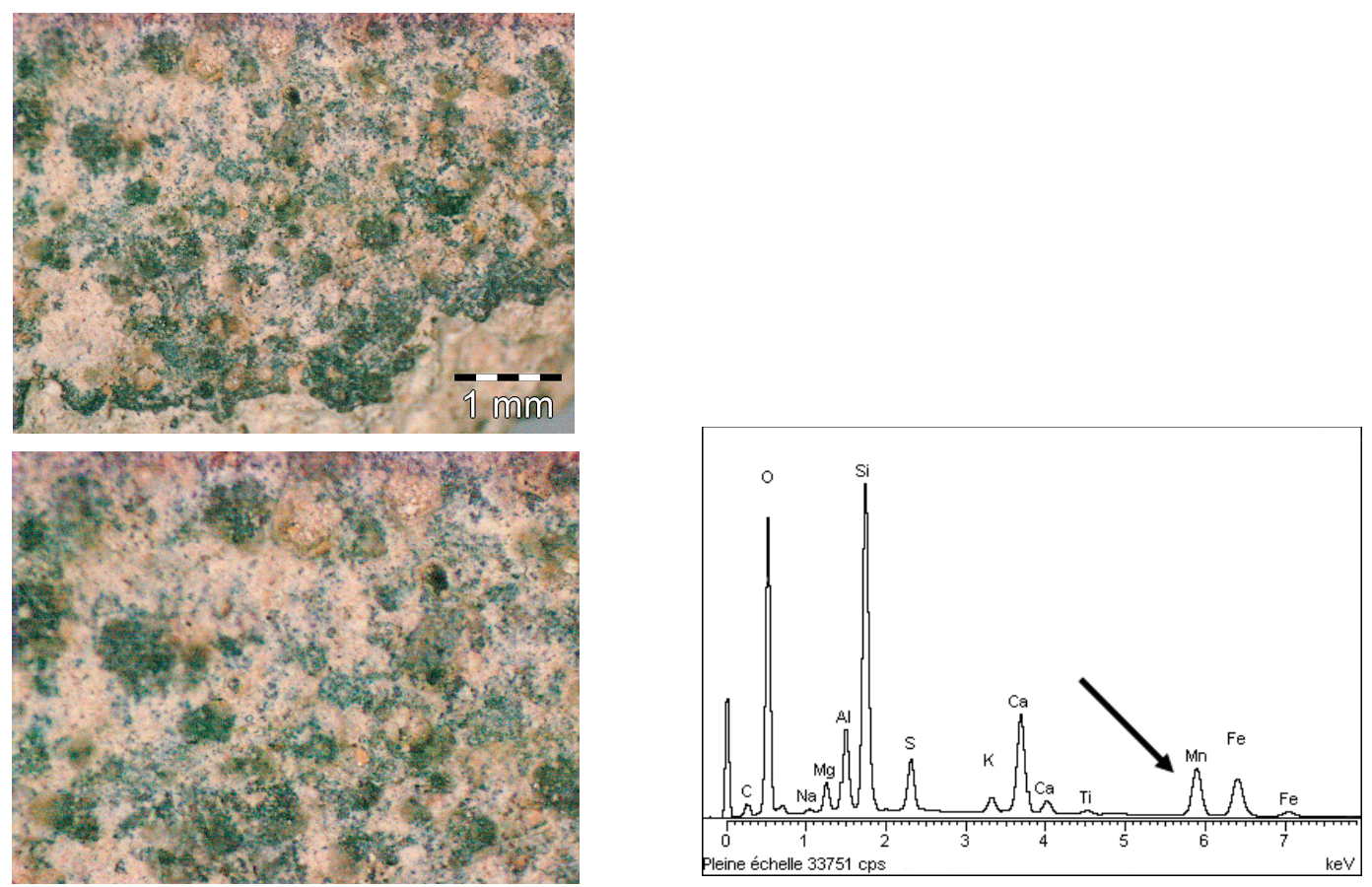

Fig. 19. - Spectres EDXS sur l'échantillon à décor « pseudo-Bichrome» (BDX 9301), montrant que pour le décor noir-brun (en haut), la matière colorante est à base de manganèse $(\mathrm{Mn})$ tandis que pour le décor noir-gris (en bas), l'élément chromogène est le fer (Fe). (CRPAA). 


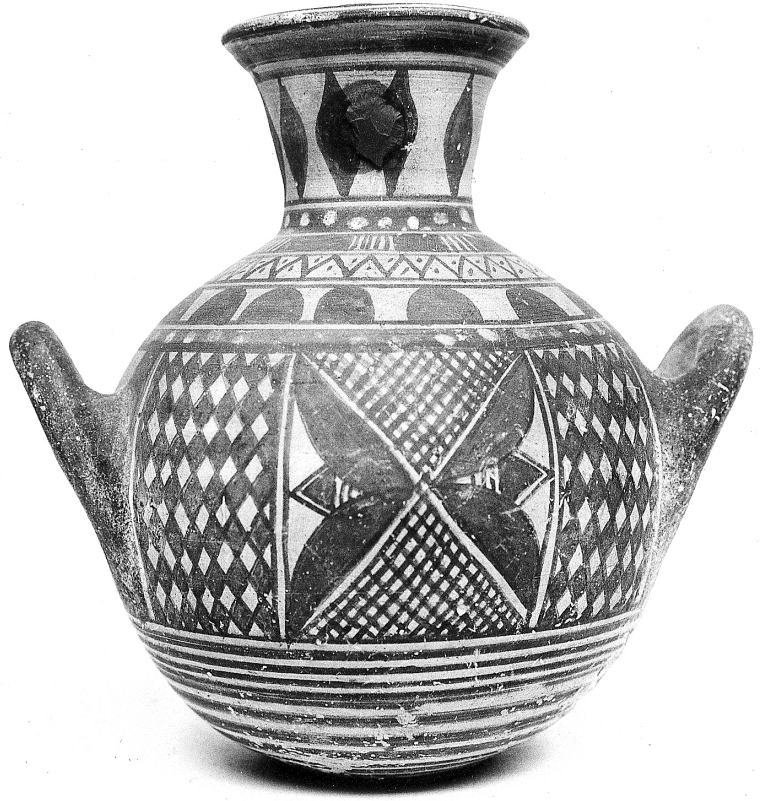

20

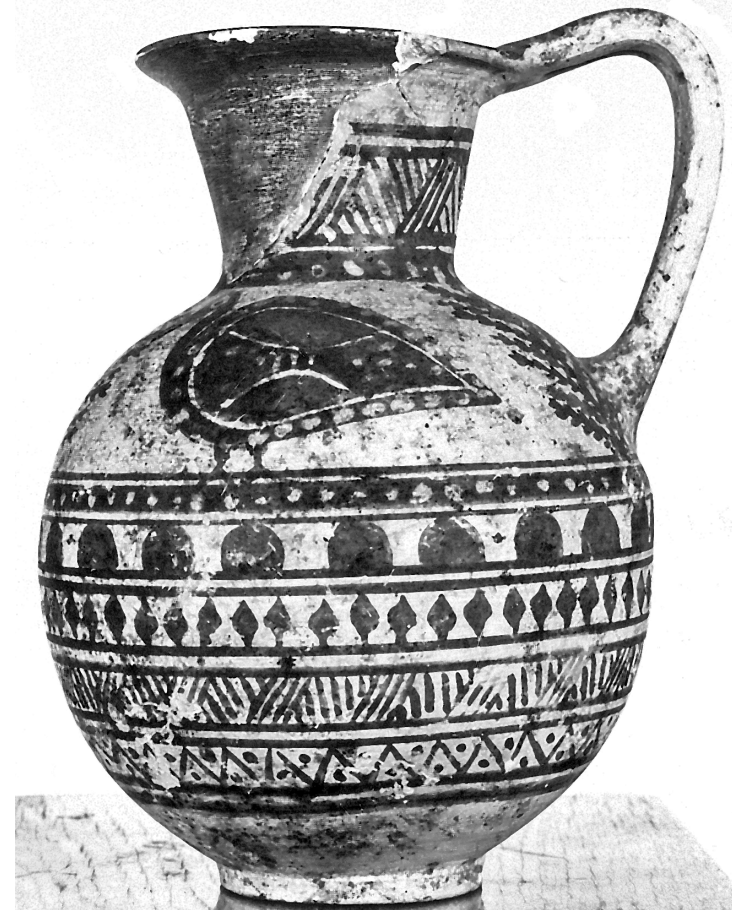

21

Fig. 20. - Amphorisque du «style d'Amathonte ». Musée du Louvre, inv. AM 188 (cliché du musée).

Fig. 21. - Cruche du « style d'Amathonte ". Musée du Louvre, inv. AM 197 (cliché du musée). L'embouchure ronde, au lieu de trilobée, est due à une restauration fautive. 

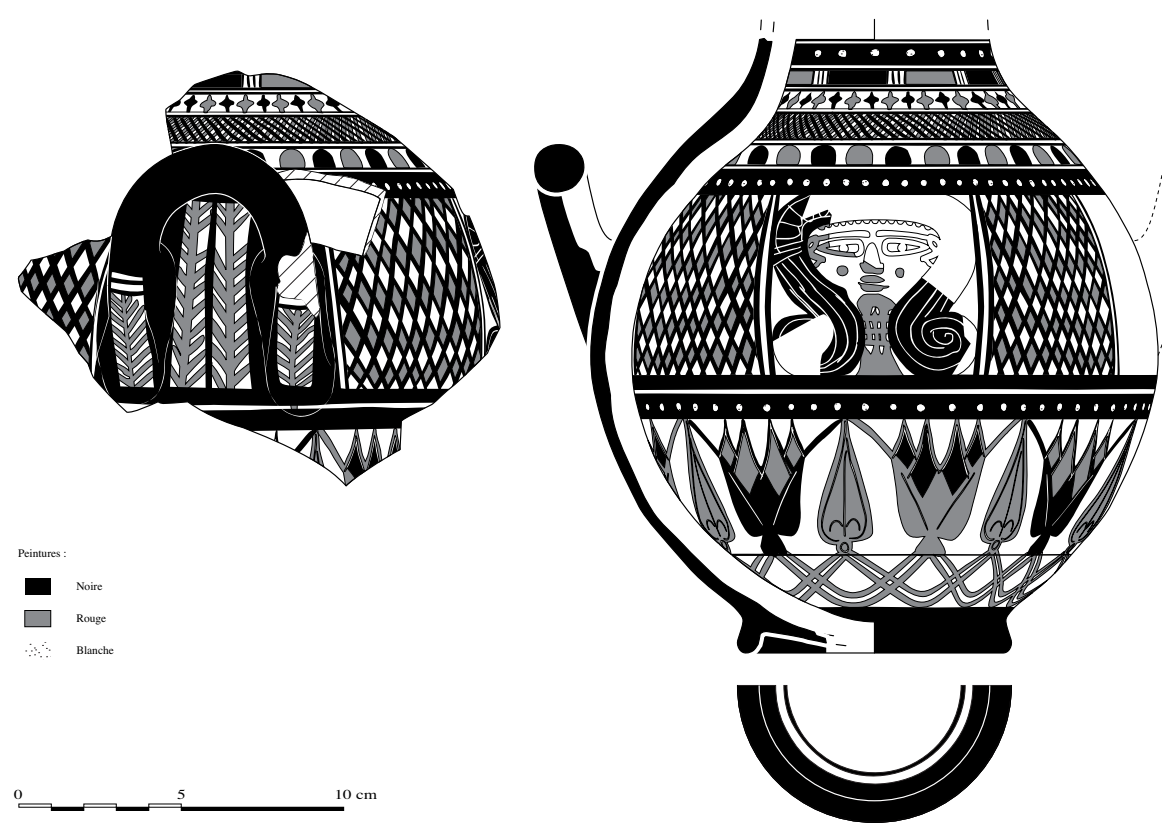

Fig. 22. - Amphorisque du «style d'Amathonte » provenant du dépôt du rempart Nord (dessin EFA, J. Humbert).
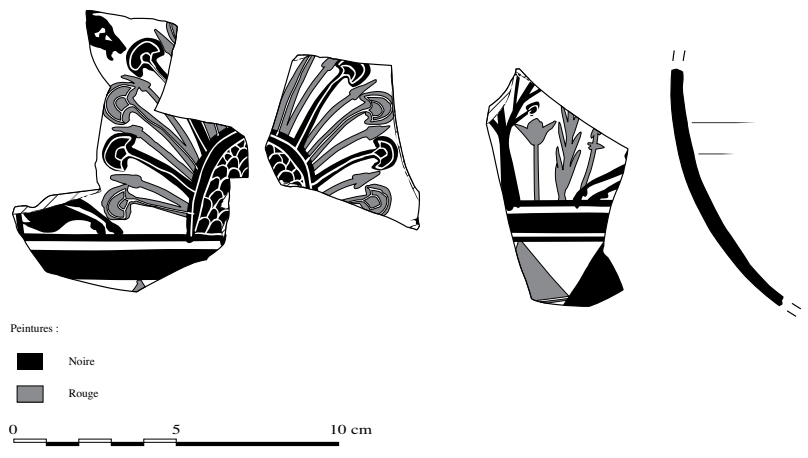

Fig. 23. - Fragment d'un amphorisque Bichrome $\mathrm{V}$ provenant du dépôt du rempart Nord (dessin EFA, J. Humbert). 


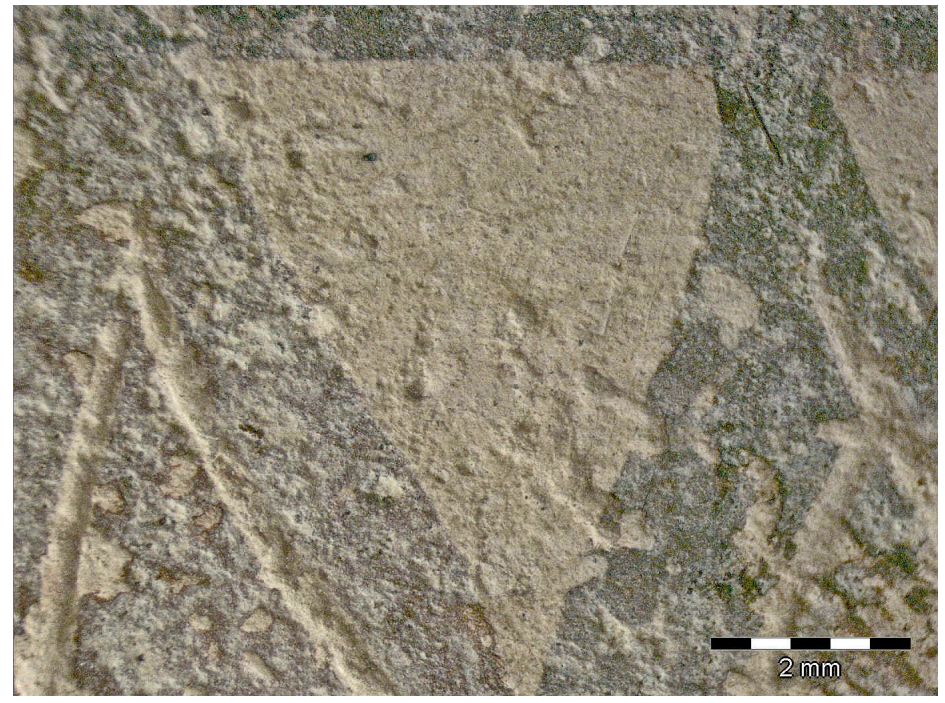

Fig. 24. - Tesson d'amphorisque du "style d'Amathonte » avec incisions (BDX 9278). Image à la loupe binoculaire en lumière naturelle rasante (cliché CRPAA).

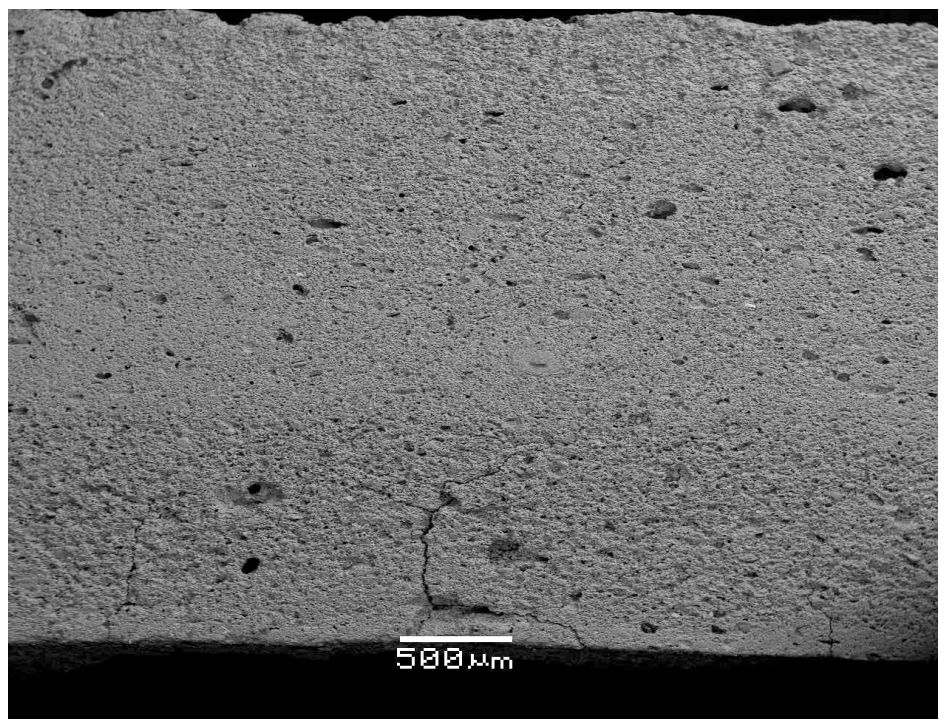

Fig. 25. - Image au microscope électronique à balayage d'un tesson d'amphorisque du «style d'Amathonte ». Agrandissement x 30, mode électrons rétrodiffusés (cliché CRPAA). 


\begin{tabular}{l|r|r|r|r|r|r|r|r}
\hline & Na2O & MgO & Al2O3 & SiO2 & K2O & CaO & TiO2 & FeO \\
\hline Moyenne & 0.6 & 3.0 & 9.7 & 35.6 & 1.1 & 44.2 & 0.7 & 5.4 \\
\hline Ecart type & 0.1 & 0.3 & 0.3 & 0.9 & 0.2 & 1.4 & 0.0 & 0.4 \\
\hline
\end{tabular}

Fig. 26. - Composition élémentaire (analyse EDS moyenne de sept mesures), exprimée en pourcentages pondéraux d'oxydes, de la terre cuite d'un amphorisque du "style d'Amathonte " (BDX 9278) (CRPAA).
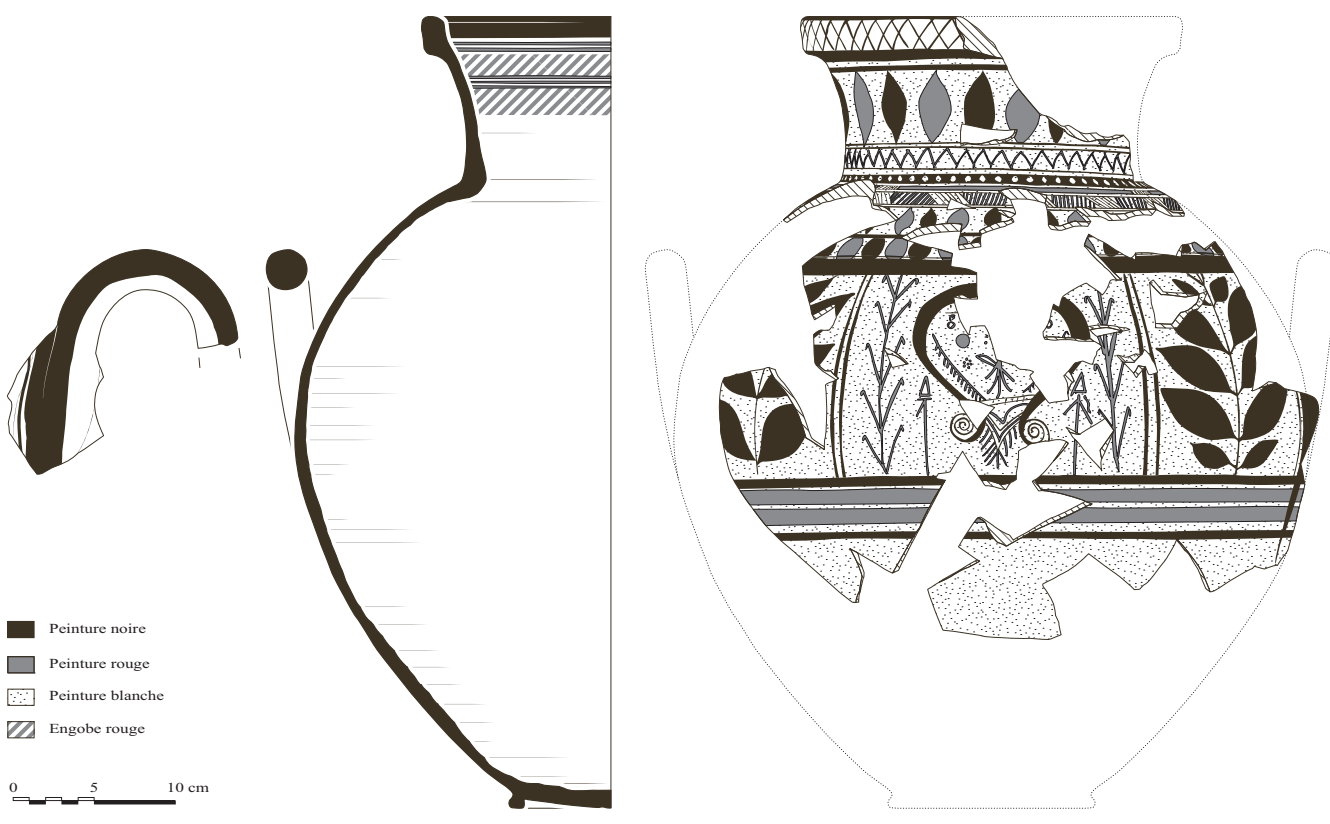

Fig. 27. - Amphore du «style d'Amathonte " provenant du dépôt du rempart Nord (dessin EFA, J. Humbert). 



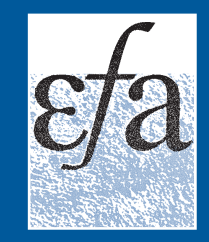

ÉCOLE FRANÇAISE D'ATHÈNES

Didotou 6 GR - 10680 Athènes www.efa.gr 